\title{
THE ENCLOSING OF CELLS IN THREE SPACE BY SIMPLE CLOSED SURFACES
}

\author{
BY \\ H. C. GRIFFITH( ${ }^{(1)}$
}

1. Introduction. A subset $X$ of Euclidean $n$-space $R^{n}$ which is homeomorphic to an Euclidean polyhedron is called tame by Fox-Artin [4]( $\left.{ }^{2}\right)$ provided there is a homeomorphism of $R^{n}$ onto itself which carries $X$ onto an Euclidean polyhedron. A question that naturally arises is under what conditions will a homeomorph of an Euclidean polyhedron be tame.

As a step toward the solution of this problem Harrold [6] shows that an arc or simple closed curve in $R^{3}$ with a certain property $P$ (defined below) has a complement which is homeomorphic to the complement of its prototype, an evident necessary condition for being tame.

In this paper an extension of the definition of property $P$ to $k$-cells in $R^{3}$, $k=1,2$, or 3 , is made, and an extension of the result of Harrold to $k$-cells is made at the cost of imposing an extra condition which is always fulfilled when $k=1$. The techniques used are those of Harrold, and depend strongly on the results of Alexander [1]. Much use is made of the concept of a semi-linear map as used by Graeub [5] and Moise [9]. As in Harrold-Moise [7], the set $K$ is called locally polyhedral at a point $p$ provided there is a neighborhood of $p$ which meets $K$ in a finite (or null) polyhedron. The set $K$ is called locally polyhedral modulo $C$ if it is locally polyhedral at each point of the complement of $C$.

2. Definitions and notation. Euclidean $k$-space will be denoted by $R^{k}$ and a fixed rectangular Cartesian coordinate system will be assumed chosen for $R^{k}$. The closure of a subset $A$ of a space $R$ will be denoted by $\mathrm{Cl}_{R} A$, or simply $\mathrm{Cl} A$ if it is clear from the context what space $R$ is meant. The boundary of $A$ in $R$ will be denoted by $B_{R}(A)$ and is defined to be $\left[\mathrm{Cl}_{R} A\right] \cap\left[\mathrm{Cl}_{R}(R \backslash A)\right]$, where $X \backslash Y$ denotes the set of points in $X$ but not in $Y$. The set $E^{k}$ is defined to be the set of all points $\left(x_{1}, \cdots, x_{k}\right)$ of $R^{k}$ such that $0 \leqq x_{i} \leqq 1$ for each $i=1, \cdots, k$, and $C^{k}$ denotes a topological image of $E^{k}$ in $R^{3}$ for $k=1,2$, or 3 . The image $C^{k}$ will be called a $k$-cell and a 0 -cell is defined to be a point. The symbol $C$ without a superscript is to be interpreted as a $k$-cell for some $k=1,2$, or 3 , and the term "a cell" as "a $k$-cell for $k=0,1,2$, or 3."

Presented to the Society, November 27, 1953; received by the editors April 5, 1954 and, in revised form, April 13, 1955.

(1) Many of the results in this paper were obtained, or are similar to results obtained, in the author's Doctoral Dissertation. This Dissertation was written at the University of Tennessee under the direction of O. G. Harrold, Jr., during the tenure of a National Science Foundation Fellowship.

(2) The numbers in brackets refer to the bibliography. 
If $h$ is any homeomorphism of $E^{k}$ onto $C$ for $k=1,2$, or 3 , that subset of $C$ which is the image under $h$ of $B_{R^{k}}\left(E^{k}\right)$ is denoted by $\partial C$, and is seen not to depend on $h$. If $C$ is a 0 -cell, $\partial C$ is defined to be the null set. An open $k$-cell is a homeomorph of $E^{k} \backslash B_{R^{k}}\left(E^{k}\right)$, and a $k$-sphere is a homeomorph of $B_{R^{k+1}}\left(E^{k+1}\right)$. A 2-cell (an open 2-cell) will occasionally be called a disk (an open disk).

If $K$ is a 2-sphere in $R^{3}$ then Int $K$ and Ext $K$ will be used to denote respectively the bounded and unbounded domains complementary to $K$ (see [11, Theorem 5.3]).

The null set will be denoted by $\square$. The symbol $\delta(A)$ denotes the diameter of the set $A$, defined as usual by $\delta(A)=\sup _{a, b} \in A d(a, b)$ where $d(a, b)$ denotes Euclidean distance. Two sets $A$ and $B$ are called separate in $R$ provided $A \cap \mathrm{Cl}_{R} B=B \cap \mathrm{Cl}_{R} A=\square$.

The topological product of two spaces $M$ and $N$ is denoted by $M \times N$. Two continuous maps $f_{0}$ and $f_{1}$ of $A$ into $B$ are said to be homotopic (in $B$ ) if there is a continuous map $F$ (called a homotopy) of $A \times E^{1}$ into $B$ which agrees with $f_{0}$ on $A \times 0$ and with $f_{1}$ on $A \times 1$. When $f_{0}$ and $f_{1}$ are homeomorphisms, then a homotopy $F$ of $f_{0}$ and $f_{1}$ is called an isotopy provided the restriction of $F$ to $A \times t$ is a homeomorphism for each $t$ in $E^{1}$.

2.11 Definition. Let $\mathfrak{S}$ denote the non-null class of all homeomorphisms of $E^{k}=E^{1} \times E^{k-1}$ onto $C$, and let

$$
\mathfrak{T}^{k}=\left\{T \mid T=h\left(x \times E^{k-1}\right) \text { for some } x \text { in } E^{1} \text { and } h \text { in } \mathfrak{S}\right\} \text {. }
$$

2.12 Definition. For each $h$ in $\mathfrak{S}$ let

$$
\mathfrak{T}_{h}^{k}=\left\{T \in \mathfrak{T}^{k} \mid T=h\left(x \times E^{k-1}\right) \text { for some } x \text { in } E^{1}\right\} .
$$

It is evident that if $T \in \mathfrak{T}^{k}$ then $C \backslash T$ is either connected or consists of exactly two components $A_{0}$ and $A_{1}$ and that $A_{i} \cup T=\mathrm{Cl}\left(A_{i}\right)$ is a $k$-cell, $i=0,1$.

It is well known that the collection of all closed subsets of $C$ forms a metric space under the Hausdorff metric [8] $\sigma$ which is defined as follows.

2.13 Definition. $\sigma(A, B)=\max \left[\sup _{a} \in A d(a, B), \sup _{b} \in B d(A, b)\right]$.

By the remark following Definition 2.12 if $T \in \mathfrak{T}^{k}$ then $C \backslash T$ may be written as $M^{\prime} \cup N^{\prime}$, where $M^{\prime}$ is a component of $C \backslash T$ and $N^{\prime}$ is either a component of $C \backslash T$ or the null set. Letting $M=M^{\prime} \cup T$ and $N=N^{\prime} \cup T, T$. determines a triple of non-null closed subsets $(T, M, N)$ of $C$. For any pair $T_{1}, T_{2}$ of elements of $\mathfrak{T}^{k}$, make the following definition.

\subsection{Definition.}

$\rho\left(T_{1}, T_{2}\right)=\sigma\left(T_{1}, T_{2}\right)+\min \left[\sigma\left(M_{1}, M_{2}\right)+\sigma\left(N_{1}, N_{2}\right), \sigma\left(M_{1}, N_{2}\right)+\sigma\left(N_{1}, M_{2}\right)\right]$.

Evidently $\rho(T, T)=0$, and if $\rho\left(T_{1}, T_{2}\right)=0$ then $\sigma\left(T_{1}, T_{2}\right)=0$ so that $T_{1}=T_{2}$. For any triple $T_{1}, T_{2}, T_{3}$ of elements of $\mathfrak{T}^{k}$ and a proper choice of notation 


$$
\rho\left(T_{1}, T_{2}\right)=\sigma\left(T_{1}, T_{2}\right)+\sigma\left(M_{1}, M_{2}\right)+\sigma\left(N_{1}, N_{2}\right),
$$

and

$$
\rho\left(T_{2}, T_{3}\right)=\sigma\left(T_{2}, T_{3}\right)+\sigma\left(M_{2}, M_{3}\right)+\sigma\left(N_{2}, N_{3}\right) .
$$

Adding these two and using the triangle inequality for $\sigma$ gives

$$
\rho\left(T_{1}, T_{2}\right)+\rho\left(T_{2}, T_{3}\right) \geqq \sigma\left(T_{1}, T_{3}\right)+\sigma\left(M_{1}, M_{3}\right)+\sigma\left(N_{1}, N_{3}\right) \geqq \rho\left(T_{1}, T_{3}\right) .
$$

Thus $\rho$ is a metric on. $\mathfrak{T}^{k}$.

The superscript of $\mathfrak{T}^{k}$ will hereafter be omitted when no loss of clarity results.

2.21 Definition. For every $T \in \mathfrak{T}$ and $\epsilon>0$ define $\mathfrak{B}(T, \epsilon)$ as the set of all $K \subset R^{3}$ satisfying the following conditions:

1. $K$ is a topological 2-sphere.

2. $T C$ Int $K$.

3. $K$ is locally polyhedral modulo $C$.

4. $K \cap C=T_{1} \cup T_{2}$, where $T_{1} \cap T_{2}=\square$, where $T_{i}=\square$ or $T_{i} \in \mathfrak{T}$ for $i=1,2$, and where if $T \subset \partial C$, then $T_{2}=\square$.

5. $K \subset S(T, \epsilon)$.

It is evident that both $\mathfrak{I}$ and $\mathfrak{P}(T, \epsilon)$ depend on the cell $C$. Since with few exceptions only one cell $C$ will be under consideration, this dependence is not indicated in the notation. However, when this is not the case, these sets will be denoted by $\mathfrak{T}(C)$ and $\mathfrak{B}(C, T, \epsilon)$ respectively.

2.22 Definition. $C$ will be said to have property $\mathcal{P}$ provided that for each $T \in \mathfrak{T}$ and $\epsilon>0$ the set $\mathfrak{B}(T, \epsilon)$ is non-null.

2.23 Definition. $C$ will be said to have property $P$ relative to a subset $\mathfrak{T}_{0}$ of $\mathfrak{T}$ provided for every $\epsilon>0$ and $T \in \mathfrak{T}_{0}$ the set $\mathfrak{B}(T, \epsilon)$ is non-null.

2.31 Definition. For every $T \in \mathfrak{T}$ and $\epsilon>0$ let $\mathfrak{D}(T, \epsilon)$ denote the set of all $D \subset R^{3}$ such that

1. $D$ is a topological 2-cell.

2. $\partial D \cap C=\square$.

3. $D \cap C \in \mathfrak{T}$.

4. $D$ is locally polyhedral modulo $C$.

5. $\rho(T, D \cap C)<\epsilon$.

6. If $C \backslash D$ has two components $C_{1}$ and $C_{2}$, then there is an $\eta>0$ such that if $N$ is a connected set meeting both $C_{1}$ and $C_{2}$ with $\delta(N)<\eta$, then $N$ meets $D$ also.

2.32 Definition. $C$ will be said to have the disk property relative to a subset $\mathfrak{I}_{0}$ of $\mathfrak{T}$ provided $\mathfrak{D}(T, \epsilon)$ is non-null for every $\epsilon>0$ and $T \in \mathfrak{T}_{0}$. If $C$ has the disk property relative to the whole set $\mathfrak{T}$, then it will simply be said that $C$ has the disk property.

2.33 Definition. $C$ will be said to have the uniform disk property relative to a subset $\mathfrak{T}_{0}$ of $\mathfrak{T}$ provided to each $\omega>0$ there corresponds $a \delta>0$ such that if $T \in \mathfrak{T}_{0}$ and $\epsilon>0$ there is a $D$ in $\mathfrak{D}(T, \epsilon)$ with $d(\partial D, C)>\delta$ and $D \subset S(T, \omega)$. 
When $C$ has the uniform disk property relative to $\mathfrak{T}_{h}$ for each $h$ in $\mathfrak{S}, C$ will be said to have the uniform disk property.

2.4 Definition. $C$ will be said to have the enclosure property provided for each $\epsilon>0$ there is a polyhedral topological 2-sphere $K$ in $S(C, \epsilon)$ with $C \subset$ Int $K$.

3. Relations between the metrics. The metric $\rho$ is chosen for $\mathfrak{T}$ in preference to the somewhat more simple metric $\sigma$ chiefly because $\sigma$ does not have the property described in this lemma.

3.1 Lemma. If $U_{1}, U_{2}$, and $T$ are elements of $\mathfrak{T}$ such that $T$ separates $U_{1}$ and $U_{2}$ on $C$, then there is a $\beta>0$ such that every element $T^{\prime}$ of $\mathfrak{T}$ with $\rho\left(T, T^{\prime}\right)$ $<\beta$ separates $U_{1}$ and $U_{2}$ on $C$.

Proof. The notation may be assumed chosen so that $M_{0} \supset U_{1}$ and $N_{0} \supset U_{2}$ where $M_{0}$ and $N_{0}$ are the components of $C \backslash T$. If $T^{\prime} \in \mathfrak{T}$ is disjoint from $U_{1}$ and $U_{2}$ and does not separate $U_{1}$ and $U_{2}$ on $C$, then both $U_{1}$ and $U_{2}$ lie in the same component $M_{0}^{\prime}$ of $C \backslash T^{\prime}$. Choose $p_{i} \in U_{i}, i=1,2$ and let $M=M_{0} \cup T$, $N=N_{0} \cup T, M^{\prime}=M_{0}^{\prime} \cup T^{\prime}$, and $N^{\prime}=N_{0}^{\prime} \cup T^{\prime}$ where $N_{0}^{\prime}$ is either null or the component of $C \backslash T^{\prime}$ other than $M_{0}^{\prime}$. Then

$$
\rho\left(T, T^{\prime}\right) \geqq \min \left[\sigma\left(M, M^{\prime}\right)+\sigma\left(N, N^{\prime}\right), \sigma\left(M, N^{\prime}\right)+\sigma\left(N, M^{\prime}\right)\right],
$$

so

$$
\rho\left(T, T^{\prime}\right) \geqq \min \left[\sigma\left(M, M^{\prime}\right), \sigma\left(N, M^{\prime}\right)\right]
$$

or

$$
\rho\left(T, T^{\prime}\right) \geqq \min \left[\sup _{p \in M^{\prime}} d(M, p), \sup _{p \in M^{\prime}} d(N, p)\right]
$$

or

$$
\rho\left(T, T^{\prime}\right) \geqq \min \left[d\left(M, p_{2}\right), d\left(N, p_{1}\right)\right] .
$$

But $p_{2} \in U_{2} \subset N$ while $p_{1} \in U_{1} \subset M$, so $\beta_{1}=\min \left[d\left(M, p_{2}\right), d\left(N, p_{1}\right)\right]>0$. Also $T \cap\left(U_{1} \cup U_{2}\right)=\square$, so $\beta_{2}=d\left(T, U_{1} \cup U_{2}\right)>0$. Hence $\beta=\min \left(\beta_{1}, \beta_{2}\right)$ is positive and independent of $T^{\prime}$. Further, if $\rho\left(T, T^{\prime}\right)<\beta$, then the assumption that $T^{\prime}$ does not separate $U_{1}$ and $U_{2}$ on $C$ leads to a contradiction.

3.2. Lemma. If $T \in \mathfrak{T}$ and $\delta>0$ then there is an $\eta>0$ such that the complement of $S(T, \delta)$ lies in Ext $K$ for every $K$ in $\mathfrak{P}(T, \eta)$.

Proof. Choose $p \in R^{3}$ and $r$ sufficiently large that $S(T, \delta) \subset S(p, r)$. Then $R=R^{3} \backslash S(p, r)$ is connected and disjoint from $S(T, \delta / n)$ for each $n=1$, $2, \cdots$, and hence determines a component $R_{n}$ of $R^{3} \backslash S(T, \delta / n)$ for each $n$. The sequence $\left\{R_{n}\right\}$ is monotone nondecreasing and has as limit $U R_{n}$, which is clearly in $R^{3} \backslash T$. But any point $x$ of $R^{3} \backslash T$ can be joined to $R$ by an arc $A_{x}$ in $R^{3} \backslash T$ and if $\delta / n$ is less than $d\left(T, A_{x} \cup R\right)$ then $A_{x}$ and hence $x$ is in $R_{n}$. Thus 
the reverse inclusion $\left(R^{3} \backslash T\right) \subset \cup R_{n}$ also holds and $U R_{n}=R^{3} \backslash T$. This means that any closed set disjoint from $T$ lies in $R_{n}$ for all sufficiently large $n$. In particular, for the assigned $\delta$ there is an $N$ such that the complement of $S(T, \delta)$ lies in $R_{N}$. Now if $\eta<d\left(T, R_{N}\right)$ and $K \in P(T, \eta)$, then $R_{N}$ is an unbounded set in the complement of $K$ and hence $R_{N} \subset$ Ext $K$. Since the complement of $S(T, \delta)$ lies in $R_{N}$, the desired $\eta$ has been found.

3.3 CoRollary. $A$ cell has property $\mathcal{P}$ (property $\mathcal{P}$ relative to $\mathfrak{T}_{0}$ ) if and only if for each $\epsilon>0$ and $T \in \mathfrak{T}\left(T \in \mathfrak{T}_{0}\right)$ there is a $K$ in $\mathfrak{P}(T, \epsilon)$ with Int $K \subset S$ $(T, \epsilon)$.

\section{Property $\Phi$ and the disk property.}

4.1. TheoRem. In order that $C$ have property $\mathcal{P}$ (property $\mathcal{P}$ relative to $\mathfrak{T}_{0}$ ) it is necessary and sufficient that for every $\epsilon>0$ and $T \in \mathfrak{T}\left(T \in \mathfrak{T}_{0}\right)$ there be a $K$ in $\mathfrak{P}(T, \epsilon)$ satisfying the following two conditions:

1. $(K \cup$ Int $K) \subset S(T, \epsilon)$.

2. If $K \cap C$ has two components, $U_{1}$ and $U_{2}$, then $T$ separates $U_{1}$ and $U_{2}$ on $C$.

Proof. The sufficiency is obvious, so suppose $C$ has property $P$ (property $P$ relative to $\left.\mathfrak{T}_{0}\right)$ and let $\epsilon>0$ and $T \in \mathfrak{T}\left(T \in \mathfrak{T}_{0}\right)$ be assigned. Two cases arise.

CASE 1. $T \cap \partial C=\partial T$. Then $T=h\left(a \times E^{k-1}\right)$ for some $h \in \mathfrak{S}$ and $0<a<1$, and $M_{1}=\mathrm{U}_{0 \leqq x<a} h\left(x \times E^{k-1}\right)$ and $M_{2}=\mathrm{U}_{a<x \leqq 1} h\left(x \times E^{k-1}\right)$ are the components of $C \backslash T$. Let $p_{i}$ be a point of $M_{i}, i=1,2$, and let $\eta=\min \left[\epsilon, d\left(T, p_{1}\right)\right.$, $\left.d\left(T, p_{2}\right)\right]$. Then $\mathfrak{B}(T, \eta) \subset \mathfrak{B}(T, \epsilon)$ since $\eta<\epsilon$, so it will suffice to show there is a $K$ in $\mathfrak{B}(T, \eta)$ meeting the requirements. Corollary 3.3 guarantees there is a $K$ in $\mathfrak{B}(T, \eta)$ with Int $K \subset S(T, \eta)$, and this, together with 5 of Definition 2.21 means that this $K$ satisfies condition 1 .

Now let $i$ be either 1 or 2 . Since $d\left(p_{i}, T\right) \geqq \eta$ and $(K \cup \operatorname{Int} K) \subset S(T, \eta)$, $p_{i}$ is in Ext $K$. So $\mathrm{Cl} M_{i}=M_{i} \cup T$ contains $p_{i} \in$ Ext $K$ and $T \subset$ Int $K$, so $K \cap C l M_{i}=K \cap\left(M_{i} \cup T\right)=K \cap M_{i} \neq \square$. Thus $K \cap M_{i}=U_{i} \in \mathfrak{T}$ by 4 of Definition 2.21. But since $T$ separates $M_{1}$ and $M_{2}$ on $C$, it must also separate $U_{1}=M_{1} \cap K$ and $U_{2}=M_{2} \cap K$ on $C$, so condition 2 is satisfied.

Case 2. $T \subset \partial C$. Then Corollary 3.3 guarantees condition 1 and condition 2 is vacuously fulfilled, for condition 4 of Definition 2.21 requires that $K \cap C$ have but one component.

4.2. Definition. Let $\mathfrak{B}^{*}(T, \epsilon)$ denote the set of all spheres of $\mathfrak{B}(T, \epsilon)$ satisfying the conclusion of Theorem 4.1.

In terms of this notation Theorem 4.1 states that $C$ has property $P$ (relative to $\mathfrak{T}_{0}$ ) if and only if $\mathfrak{B}^{*}(T, \epsilon)$ is non-null for every $T \in \mathfrak{T}\left(T \in \mathfrak{T}_{0}\right)$ and $\epsilon>0$.

4.3. Lemma. If $T \in \mathfrak{T}$ and $\epsilon>0$, then there is $a \beta>0$ such that whenever $K \in \mathfrak{B}^{*}(T, \beta)$ and $T_{1}$ is a component of $K \cap C$, then $\rho\left(T, T_{1}\right)<\epsilon$. 
Proof. Let $\epsilon>0$ and $T \in \mathfrak{T}$ be assigned. Then $C$ may be written as $C_{1} \cup C_{2}$ where $T=h\left(x_{0} \times E^{k-1}\right)$ for some $h \in \mathfrak{S}$ and $C_{1}$ and $C_{2}$ are the images under $h$ of $\bigcup_{0 \leqq x \leqq x_{0}} h\left(x \times E^{k-1}\right)$ and $\bigcup_{x_{0} \leqq x \leqq 1} h\left(x \times E^{k-1}\right)$ respectively. Each $C_{\boldsymbol{i}}$ is a cell (one is of dimension $k$ and the other of dimension $k$ or $k-1$ according as $T \cap \partial C$ is $\partial T$ or $T$ ) and is therefore uniformly locally connected. That is, for $i=1$ and $i=2$ there is an $\omega_{i}>0$ such that if $t \in T$ and $p \in C_{i}$ with $d(t, p)<\omega_{i}$ then some connected subset $Q$ of $C_{i}$ with $\delta(Q)<\epsilon / 4$ contains both $t$ and $p$.

If $C_{i} \neq T$, straightforward application of the uniform continuity of $h$ produces an $x_{i} \neq x_{0}$ meeting the following conditions:

$4.31 T_{i}^{\prime}=h\left(x_{i} \times E^{k-1}\right)$ is a subset of $C_{i}$.

$4.32 d\left(t, T_{i}^{\prime}\right)<\omega_{i}$ for all $t \in T$.

$4.33 d[h(x, y), T]+d\left[h(x, y), T_{i}^{\prime}\right]<\epsilon / 4$ for all $x$ between $x_{i}$ and $x_{0}$ and any $y$ in $E^{k-1}$.

For $i=1,2$ let $\beta_{i}=\min \left[d\left(T, T_{i}^{\prime}\right), \epsilon / 4\right]$ or $\beta_{i}=1$ according as $C_{i} \neq T$ or $C_{i}=T$, and let $\beta=\min \left(\beta_{1}, \beta_{2}\right)$.

Suppose now that $K \in \mathfrak{P}^{*}(T, \beta)$ and that $T_{1}$ is a component of $K \cap C$. The inclusion $T_{1} \subset C_{1}$ is assumed as a notational convenience. If $t$ is any point of $T$ then by 4.32 there is a $t_{1}^{\prime} \in T_{1}^{\prime}$ with $d\left(t, t_{1}^{\prime}\right)<\omega_{1}$ and hence a connected subset $Q$ of $C_{1}$ with $\delta(Q)<\epsilon / 4$ which contains both $t$ and $t_{1}^{\prime}$. But $T \subset$ Int $K$ and, since $K \cup$ Int $K \subset S(T, \beta)$ and $d\left(T, T_{1}^{\prime}\right)>\beta, T_{1}^{\prime} \subset$ Ext $K$. The set $Q$ therefore meets both Int $K$ and Ext $K$, and must meet $K$, so $t_{1} \in Q \cap K$ may be chosen. That $K \cap C_{1}=T_{1}$ follows from condition 2 of Theorem 4.1 and the choice of the sets $C_{1}$ and $C_{2}$, so since $Q \cap K \subset C_{1} \cap K, t_{1}$ is a point of $T_{1}$. Then $d\left(t, T_{1}\right)$ $\leqq d\left(t, t_{1}\right) \leqq \delta(Q) \leqq \epsilon / 4$. But $t$ was arbitrary in $T$ so $\sup _{t \in T} d\left(t, T_{1}\right) \leqq \epsilon / 4$. Since $T_{1} \subset K \subset S(T, \beta)$, it follows that $\sup _{t_{1} \in T_{1}} d\left(T, t_{1}\right)<\beta \leqq \epsilon / 4$. Combining these yields $\sigma\left(T, T_{1}\right) \leqq \epsilon / 4$.

Now let $\left(T, C_{1}, C_{2}\right)$ and $\left(T_{1}, M, N\right)$ be the triples of sets in the expression for $\rho\left(T, T_{1}\right)$ where $N$ contains $T$. Since $C_{2}$ is connected and $T_{1} \subset C_{1}$, this requires $C_{2} \subset N$ and $M \subset C_{1}$, so that $\sup _{p \in M} d\left(p, C_{1}\right)=\sup _{q \in C_{2}} d(N, q)=0$. This means that

$$
\rho\left(T, T_{1}\right) \leqq \sigma\left(T, T_{1}\right)+\sup _{p \in C_{1}} d(M, p)+\sup _{q \in N} d\left(q, C_{2}\right) .
$$

But if $p \in C_{1}$, then $p=h(x, y)$ where $0 \leqq x \leqq x_{0}$ and $y \in E^{k-1}$. If $x<x_{1}$ then $p \in M$ so $d(M, p)=0$, and if $x_{1} \leqq x \leqq x_{0}$, then, by 4.33 together with the fact that the choice of $\beta$ implies $T_{1}^{\prime} \subset M, d(M, p) \leqq d\left(T_{1}^{\prime}, p\right) \leqq \epsilon / 4$. Similarly, if $q \in N$ then $q=h(x, y)$ with $x_{1} \leqq x \leqq 1$ so either $x_{0} \leqq x$, in which case $q \in C_{2}$ and $d\left(q, C_{2}\right)=0$, or $x_{1} \leqq x \leqq x_{0}$, so that by $4.33, d\left(q, C_{2}\right) \leqq d(q, T)<\epsilon / 4$. Combining, $\rho\left(T, T_{1}\right) \leqq \epsilon / 4+\epsilon / 4+\epsilon / 4<\epsilon$.

4.4. Theorem. If $C$ has property $\mathcal{P}$ (relative to $\mathfrak{T}_{h}$ ), then $C$ has the disk property (relative to $\mathfrak{T}_{h}$ ).

Proof. Let $T \in \mathfrak{T}\left(T \in \mathfrak{T}_{h}\right)$ and $\epsilon>0$ be assigned. Then $C \backslash T$ may be written as $N_{1} \cup N_{2}$, where $N_{1}$ is a component and $N_{2}$ is either a component or null. 
Since $T=h\left(x \times E^{k-1}\right)$ for some $h \in \mathfrak{S}$ (the given $h$ ) and some $x N_{1} \cup T$ is a $k$-cell. Choose $p_{1} \in N_{1}$ and let $\beta_{1}=d\left(p_{1}, T\right)$. By Lemma 4.3 there is a $\beta_{2}>0$ such that if $K \in \mathfrak{B}^{*}\left(T, \beta_{2}\right)$ and $T_{1}$ is a component of $K \cap C$, then $\rho\left(T, T_{1}\right)<\epsilon$. Let $\beta=\min \left(\beta_{1}, \beta_{2}\right)$ and choose $K \in \mathfrak{B}^{*}(T, \beta)$.

Now $K \cap C$ has either a single component $T_{1}$ or two components $T_{1}$ and $T_{2}$ according as $N_{2}$ is null or not, and this together with the choice of $\beta$ and condition 1 of Theorem 4.1 guarantees that $K \cap M_{1} \neq \square$ so that $K \cap M_{1}$ $=T_{1} \in \mathfrak{T}$ is but a notational convenience.

If $T_{2} \neq \square$, then $T_{2} \in \mathfrak{T}$. $K$ is then a topological 2-sphere with $T_{1}$ and $T_{2}$ a pair of disjoint $(k-1)$-cells on $K . K$ is, then, the image of the unit spherical surface $W$ under a homeomorphism $g$ which maps $V$ onto $T_{1}$ where $V$ is the point $(1,0,0)$, or the set $\left\{(x, y, 0) \in R^{3} \mid x^{2}+y^{2}=1\right.$ and $\left.x \geqq 0\right\}$, or the set $\left\{(x, y, z) \in R^{3} \mid x^{2}+y^{2}+z^{2}=1\right.$ and $\left.x \geqq 0\right\}$, according as $C$ is a 1-cell, a 2-cell, or a 3-cell. The set $g^{-1}\left(T_{2}\right)$ is disjoint from $V$ so for some $\alpha>0,\{\mathrm{Cl}[W \cap S$ $(V, \alpha)]\} \cap\left\{g^{-1}\left(T_{2}\right)\right\}=\square$. If $X=W \cap S(V, \alpha)$, then $\partial X$ is a simple closed curve separating $g^{-1}\left(T_{2}\right)$ and $V=g^{-1}\left(T_{1}\right)$ on $W$. Then $S=g(\partial X)$ is a simple closed curve separating $T_{1}$ and $T_{2}$ on $K$.

If $T_{2}$ is null $S$ may be chosen to be any simple closed curve on $K \backslash C$. In either case then, the curve $S$ divides $K$ into two closed disks, one of which meets $C$ at $T_{1}$ and at no other points. Call this disk $D$. It will now be shown that $D, T$, and $\epsilon$ have the six properties of Definition 2.31 .

1. $D$ is a topological 2-cell by the Schoenflies theorem.

2. $\partial D=S$ and $S \subset K \backslash C$ so $\partial D \cap C=\square$.

3. $D \cap C=T_{1} \in \mathfrak{T}$.

4. $D \subset K$ so $D$ is locally polyhedral $\bmod C$ by 3 of Definition 2.21 .

5. $\rho(T, D \cap C)=\rho\left(T, T_{1}\right)<\epsilon$ by choice of $\beta$ and $K$.

6. Since $K \cap N_{1}=T_{1}$ and $K$ separates $p \in$ Ext $K$ from $T \subset$ Int $K$ on $N_{1} \cup T,\left(N_{1} \cup T\right) \backslash T_{1}$ may be written as the union of $C_{1}=N_{1} \cap$ Ext $K$ containing $p$ and $C_{2}=\left(N_{1} \cup T\right) \cap$ Int $K$ containing $T$. Since $\left(\mathrm{Cl} N_{1}\right) \cap\left(\mathrm{Cl} N_{2}\right)$ is null or $T$ according as $N_{2}$ is null or not, it follows that $C_{1}$ and $N_{2}$ are separate so that $T_{1} \cap \mathrm{Cl} N_{2}=\square$ and the two components of $C \backslash T_{1}$ must be $C_{1}$ and $C_{2} \cup N_{2}$. Thus $\omega>0$ can be taken less than $d\left[T_{1}, N_{2} \cup(K \backslash D)\right]$ so that $N_{2} \cap S\left(T_{1}, \omega\right)=\square$ and $K \cap S\left(T_{1}, \omega\right)=D \cap S\left(T_{1}, \omega\right)$.

It is asserted that there is then an $\eta>0$ such that if $M$ is a connected set meeting both $C_{1}$ and $C_{2} \cup N_{2}$ with $\delta(M)<\eta$, then $M \subset S\left(T_{1}, \omega\right)$. For if this is not the case for $i=1,2, \ldots$ there is a connected set $M_{i}$ with $\delta\left(M_{i}\right)<1 / i$ and a triple of points, $p_{i} \in M_{i} \cap C_{1}, q_{i} \in M_{i} \cap\left(C_{2} \cup N_{2}\right)$ and $r_{i} \in M_{i}$ with $d\left(r_{i}, T_{1}\right)>\omega$. By a standard procedure a subsequence of indices $k_{1}$, $k_{2}, \cdots$ can be chosen such that $\left\{p_{k_{i}}\right\},\left\{q_{k_{i}}\right\}$ and $\left\{r_{k_{i}}\right\}$ all converge, and it is readily seen that they must all have the same limit point $q$. As the limit of $\left\{p_{k_{i}}\right\} \subset C_{1}$ and $\left\{q_{k_{i}}\right\} \subset C_{2} \cup N_{2}, q$ must lie in $\left(\mathrm{Cl} C_{1}\right) \cap \mathrm{Cl}\left(C_{2} \cup N_{2}\right)=T_{1}$, contradicting the fact that as the limit of $\left\{r_{k_{i}}\right\}, d\left(q, T_{1}\right) \geqq \omega$. Hence the asserted $\eta$ exists, and is the number required to fulfil condition 6 . For suppose $M$ is 
connected, $\delta(M)<\eta$, and $M$ meets both $C_{1}$ and $C_{2} \cup N_{2}$. Then $M \subset S\left(T_{1}, \omega\right)$ so $M \cap N_{2} \subset S\left(T_{1}, \omega\right) \cap N_{2}=\square$ and $M$ meets both $C_{1} \subset$ Ext $K$ and $C_{2} \subset$ Int $K$. This requires that $M$ meet $K$ and $M \cap K \subset K \cap S\left(T_{1}, \omega\right)=D \cap S\left(T_{1}, \omega\right)$.

Thus $D \in \mathfrak{D}(T, \epsilon) \neq \square$, and $C$ has the disk property (relative to $\mathfrak{I}_{h}$ ).

\section{The interior radii.}

5.1. TheOREM. If $\mathfrak{I}_{0}$ is any compact subset of $\mathfrak{I}$ and $C$ has property $\mathbb{P}$ relative to $\mathfrak{I}_{0}$, then for each $\epsilon>0$ there is a $\gamma>0$ such that for every $T \in \mathfrak{T}_{0}$ the inequality $\sup _{K \in \mathfrak{P}^{*}(T, e)} d(T, K)>\gamma$ holds.

Proof. Let $\gamma(T, \epsilon)$ denote the supremum in the statement of the theorem. If the conclusion is false, then for each integer $n$ there is a $T_{n}$ in $\mathfrak{T}_{0}$ such that $\gamma\left(T_{n}, \epsilon\right) \leqq 1 / n$. Since $\mathfrak{I}_{0}$ is compact, some subsequence $\left\{T_{n_{i}}\right\}$ converges to $T_{0} \in \mathfrak{I}_{0}$, and to simplify the notation it will be assumed that $\left\{T_{n}\right\}$ converges to $T_{0}$. Now $\mathfrak{B}^{*}\left(T_{0}, \epsilon / 2\right)$ is non-null from the hypothesis that $C$ has property $\mathcal{P}$ relative to $\mathfrak{I}_{0}$ and Theorem 4.1 , so choose $K \in \mathfrak{B}^{*}\left(T_{0}, \epsilon / 2\right)$.

For any fixed $i$ there is a $p \in T_{i}$ and a $q \in K$ such that

$$
d(p, q)=d\left(T_{i}, K\right) \text {. }
$$

So for any $r \in T_{0}, d(p, q)+d(p, r) \geqq d(q, r)$, or

$$
d\left(T_{i}, K\right) \geqq d(q, r)-d(p, r) .
$$

Since $T_{0} \subset$ Int $K, d\left(T_{0}, K\right)=2 \omega>0$, and $d(q, r) \geqq d\left(T_{0}, K\right)$, so 5.12 yields

$$
d\left(T_{i}, K\right) \geqq 2 \omega-d(p, r) .
$$

But $r$ was arbitrary in $T_{0}$, so

$$
d\left(T_{i}, K\right) \geqq 2 \omega-\inf _{r \in T_{0}} d(p, r)=2 \omega-d\left(p, T_{0}\right),
$$

and, since $\rho\left(T_{i}, T_{0}\right) \geqq d\left(p, T_{0}\right), d\left(T_{i}, K\right) \geqq 2 \omega-\rho\left(T_{i}, T_{0}\right)$. But $\left\{T_{i}\right\}$ converges to $T_{0}$ so there is an $M_{1}$ such that for all $i>M_{1}, \rho\left(T_{i}, T_{0}\right)<\omega$. Combining these facts,

$$
i>M_{1} \text { implies } d\left(T_{i}, K\right) \geqq 2 \omega-\omega=\omega .
$$

Now if $x \in R^{3}$ is such that $d\left(x, T_{0}\right)<\omega$, since $d\left(T_{0}, K\right)=2 \omega$, then $x \in \operatorname{Int} K$. But if $i>M_{1}, \rho\left(T_{i}, T_{0}\right)<\omega$ so $d\left(x, T_{0}\right)<\omega$ for every $x$ in $T_{i}$, i.e.,

$$
i>M_{1} \text { implies } T_{i} \subset \text { Int } K \text {. }
$$

Let $q$ be any point of $K \cup \operatorname{Int} K$. Since $K \cup \operatorname{Int} K \subset S\left(T_{0}, \epsilon / 2\right)$, there is a point $r$ of $T_{0}$ such that $d(r, q)<\epsilon / 2$. Corresponding to this $r$ there is a $p_{i} \in T_{i}$ such that $d\left(r, p_{i}\right)=d\left(r, T_{i}\right) \leqq \rho\left(T_{0}, T_{i}\right)$. Then $d\left(p_{i}, q\right) \leqq d\left(p_{i}, r\right)+d(r, q)$ $\leqq \rho\left(T_{0}, T_{i}\right)+\epsilon / 2$. But there is an $M_{2}$ such that if $i>M_{2}$ then $\rho\left(T_{0}, T_{i}\right)<\epsilon / 2$. For such $i$ the above inequality is $d\left(r, p_{i}\right) \leqq \epsilon / 2+\epsilon / 2=\epsilon$. So, given $q$ arbitrary in $K \cup$ Int $K$ and $i>M_{2}$ there is a $p_{i} \in T_{i}$ such that $d\left(q, p_{i}\right)<\epsilon$. The point $p_{i}$ 
depends on $q$, but the $M_{2}$ depends only on the convergence of $\left\{T_{i}\right\}$ to $T_{0}$. So

$$
i>M_{2} \text { implies } K \cup \operatorname{Int} K \subset S\left(T_{i}, \epsilon\right) \text {. }
$$

Suppose now that $K \cap C=U_{1} \cup U_{2}$ where $U_{1}$ and $U_{2}$ are in $\mathfrak{T}$. Then since $K \in P^{*}\left(\mathfrak{T}_{0}, \epsilon / 2\right)$, condition 2 of Theorem 4.1 requires that $T_{0}$ separate $U_{1}$ and $U_{2}$ on $C$. By Lemma 3.1, if $\rho\left(T_{i}, T_{0}\right)$ is less than some fixed $\beta>0, T_{i}$ also separates $U_{1}$ and $U_{2}$ on $C$, so there is an $M_{3}$ such that this is the case for all $i>M_{3}$. If $K \cap C$ does not have two components take $M_{3}=1$. In any case then

$$
i>M_{3} \text { implies when } K \cap C \text { has two components }
$$

they are separated on $C$ by $T_{i}$.

Now let $M=\max \left(M_{1}, M_{2}, M_{3}\right)$, and suppose $i>M$. Then $K$ satisfies conditions 1,3 , and 4 of Definition 2.21 for the set $\mathfrak{B}\left(T_{i}, \epsilon\right)$ by virtue of being in $\mathfrak{B}^{*}\left(T_{0}, \epsilon / 2\right)$, since these conditions do not involve the $T$ and $\epsilon$. But 5.15 and 5.16 are valid since $i>M$, so conditions 2 and 5 of Definition 2.21 are also satisfied and $K \in \mathfrak{P}\left(T_{i}, \epsilon\right)$. Conditions 1 and 2 of Theorem 4.1 are both fulfilled by $K, T_{i}$, and $\epsilon$ by virtue of 5.16 and 5.17. Thus $i>M$ implies $K \in \mathfrak{P}^{*}$ $\left(T_{i}, \epsilon\right)$.

But then $\gamma\left(T_{i}, \epsilon\right) \geqq d\left(T_{i}, K\right)$, so from $5.14, i>M$ implies $\gamma\left(T_{i}, \epsilon\right)>\omega$. Since $\gamma\left(T_{i}, \epsilon\right)<1 / i$ by choice, this is a contradiction and the theorem is established.

5.2. THEOREM. $\mathfrak{T}_{h}$ is an arc in $\mathfrak{T}$ for every $h$ in $\mathfrak{S}$.

Proof. Define $\phi: E^{1} \rightarrow \mathfrak{T}_{h}$ bv

$$
\phi(x)=h\left(x \times E^{k-1}\right) \equiv{ }_{d} T_{x} .
$$

It is obvious that $\phi$ is $1-1$ and onto. Let $x_{1} \in E^{1}$ and $\omega>0$ be assigned. Since $h$ is uniformly continuous, there is a $\theta>0$ such that whenever $\left|x_{2}-x_{1}\right|<\theta$, then $d\left[h\left(x_{1}, y\right), h\left(x_{2}, y\right)\right]<\omega / 3$ for all $y$ in $E^{k-1}$. Let $M_{i}$ be the set $\{h(x, y) \mid 0$ $\left.\leqq x \leqq x_{i}\right\}$, and $N_{i}$ be $\left\{h(x, y) \mid x_{i} \leqq x \leqq 1\right\}, i=1,2$. Then $\rho\left(T_{1}, T_{2}\right)=\sigma\left(T_{1}, T_{2}\right)$ $+\sigma\left(M_{1}, M_{2}\right)+\sigma\left(N_{1}, N_{2}\right)$, and an elementary calculation shows each of the terms on the right is less than $\omega / 3$. Thus $\phi$ is a continuous 1-1 map of a compact space onto a Hausdorff space and must be topological, so $\mathfrak{T}_{h}$ is an arc.

5.3. Corollary. If $C$ has property $\mathcal{P}$ relative to $\mathfrak{T}_{h}$ and $T_{x}$ denotes the $(k-1)$-cell $h\left(x \times E^{k-1}\right)$, then for each $\epsilon>0$ there is a $\gamma>0$ such that for all $x$ in $E^{1}$ the inequality $\gamma\left(T_{x}, \epsilon\right)>\gamma$ holds.

6. The construction lemmas. Let $h$ be a fixed homeomorphism of $E^{k}$ onto $C$ and let $a$ be a point of $T_{0} \backslash \partial T_{0}$, where $T_{x}$ denotes $h\left(x \times E^{k-1}\right), 0 \leqq x \leqq 1$. Since $a \in \mathrm{Cl}\left(R^{3} \backslash C\right)$, well known results in the theory of accessibility assure that there is an arc $A^{\prime}$ from a point $a_{1}$ to $a$ which meets $C$ only at $a$. If $A^{\prime \prime}$ is a topological ray in $R^{3} \backslash C$ with initial point $a_{1}$, then $A^{\prime} \cup A^{\prime \prime}$ contains a topological ray $A$ which meets $C$ only at its initial point $a$. 
Similarly a topological ray $B$ meeting $C$ only at its initial point $b \in T_{1} \backslash \partial T_{1}$ can be chosen, and the two rays $A$ and $B$ may be taken disjoint and locally polyhedral modulo $C$.

A partial order on the elements of $\mathfrak{T}$ is now defined by $T<U$ provided both the conditions $T$ separates $A$ and $U$ on $A \cup C \cup B$ and $U$ separates $T$ and $B$ on $A \cup C \cup B$ are met. This order is extended to the set consisting of all elements of $T$ and all points of $A \cup B$ by $U<T$ and $T<V$ for every choice of $U \in A, V \in B$, and $T$ an element of $\mathfrak{T}$ which separates $A$ and $B$ on $A \cup C \cup B$. For each $T$ in the set on which this order is defined it will be convenient to let $A(T)$ and $B(T)$ denote the components of $(A \cup C \cup B) \backslash T$ containing an unbounded subset of $A$ and an unbounded subset of $B$ respectively. It is easily verified that $T<U$ holds if and only if both $T \cup \mathcal{A}(T) \subset \mathcal{A}(U)$ and $\mathrm{B}(T) \supset U \cup \mathrm{B}(U)$.

It should also be noted that for elements of $\mathfrak{T}$ the order relation depends only on the choice of $a \in T_{0} \backslash \partial T_{0}$ and $b \in T_{1} \backslash \partial T_{1}$, and not on the choice of rays $A, B$, from $a, b$. In the remainder of this section $h, A$, and $B$ will be assumed chosen and fixed.

6.1. Lemma. Let $D, K_{1}, T$ and $\epsilon>0$ be related as follows:

1. $T \in \mathfrak{T}$ and separates $A$ and $B$ on $A \cup C \cup B$.

2. $K \in \mathfrak{P}^{*}(T, \epsilon)$.

3. $K \cap C=U_{1} \cup U_{2}$ where both $U_{1}$ and $U_{2}$ are in $\mathfrak{T}$.

4. Either $U_{1}<T<U<U_{2}$ or $U_{1}<U<T<U_{2}$, where $D \cap C=U \in \mathfrak{T}$.

5. $D \in \mathfrak{D}(U, \omega)$ for every $\omega>0$.

6. $\left(D \cup K_{1}\right) \cap(A \cup B)=\square$.

7. $d(\partial D, C)>\epsilon$.

Then there is a $K_{2}$ in $\mathfrak{B}^{*}(T, \epsilon)$ with $K_{2} \cap C$ either $U_{1} \cup U$ or $U \cup U_{2}$ according as $T<U$ or $U<T$.

Proof. Suppose $U_{1}<T<U<U_{2}$. Then these four sets are pairwise disjoint and $U$ separates $U_{1}$ and $U_{2}$ on $C$. Since $U \in \mathfrak{T}$, there is a $g \in \mathfrak{E}$ such that $U=g\left(x \times E^{k-1}\right)$ for some $x$ with $0<x<1$, for neither $g\left(0 \times E^{k-1}\right)$ nor $g\left(1 \times E^{k-1}\right)$ can separate $U_{1}$ and $U_{2}$. Consequently $C \backslash U=\mathcal{A} \cup B$ where $\mathcal{A}$ contains $U_{1}$ and $T, B$ contains $U_{2}$, and $A \cup U$ is a $k$-cell. An arc $E$ in $A \cup U$ with one endpoint in $U$, the other in $U_{1}$ and otherwise disjoint from $U \cup U_{1}$ can be constructed. Since $T$ separates $U_{1}$ and $U$ on $C, E$ meets $T$ so a sub-arc $E^{\prime}$ of $E$ meeting both $T$ and $U$ and not meeting $U_{1}$ can be found. Then $T \cup U \cup E^{\prime}$ is a connected set in the complement of $K_{1}$ so since $T \subset$ Int $K_{1}, U$ also is in Int $K_{1}$.

Thus $D \cap$ Int $K_{1} \neq \square$, and since $K_{1} \cup$ Int $K_{1} \subset S(T, \epsilon) \subset S(C, \epsilon)$ while $d(\partial D, C)>\epsilon, D \cap$ Ext $K_{1} \neq \square$ so that $K_{1} \cap D \neq \square$. Since $D \cap C \cap K_{1}=U \cap K_{1}$ and $U C$ Int $K_{1}, D \cap C \cap K_{1}=\square$ so both $D$ and $K_{1}$ are locally polyhedral in some neighborhood $V$ of $D \cap K_{1}$ containing no points of $C \cup \partial D$, and $V$ may be taken disjoint from $A \cup B$ also by condition 6 of the hypothesis. By shifting 
the vertices of $D$ which lie in $V$ a distance so small that no point of $D$ outside $V$ is moved, $D$ and $K_{1}$ may be brought into relative general position. Then $D \cap K_{1}$ will consist of a finite collection of mutually disjoint simple closed curves $s_{1}, s_{2}, \cdots, s_{n}$.

Each $s_{k}$ bounds a unique sub-disk $D_{k}$ of $D$ and a pair of disks $X_{k}$ and $Y_{k}$ on $K_{1}$, where $Y_{k}$ contains $U_{1}$. An arc $c$ of $C$ can be chosen so that $c$ meets $\partial C$ only at its two end points $a$ and $b$. Then $A \cup c \cup B$ is a topological line and hence a continuous 1-cycle, so the theory of linkages may be applied to $A \cup c \cup B$ and the curves $s_{k}$. The arc $c$ may be chosen so that it meets each of the sets $U_{1}, U$, and $U_{2}$ in a single point, so that for any choice of $k$ either $X_{k} \cap C=U_{2}, Y_{k} \cap C=U_{1}$ and $D_{k} \cap C=U$ or $X_{k} \cap C=\square, Y_{k} \cap C=U_{1} \cup U_{2}$, and $D_{k} \cap C=\square$ according as $s_{k}$ links $A \cup c \cup B$ or not. Thus if any $s_{k}$ fails to link $A \cup c \cup B$, the corresponding $X_{k}$ is a sub-disk of $K_{1} \backslash\left(U_{1} \cup U_{2}\right)$ and an index $j$ can be found so that $X_{j} \cap D=s_{j}$. For this $j$ the set $\left(D \backslash D_{j}\right) \cup X_{j}$ is a disk, and a neighborhood $V$ of $X_{j}$ can be chosen so that $V \cap(A \cup C \cup B)=\square$ and $V \cap D \cap K_{1} \subset X_{j}$. Then $\left(D \backslash D_{j}\right) \cup X_{j}$ can be deformed away from $X_{j}$ semilinearly so that no point outside $V$ is moved, and the resulting disk $D^{*}$ has the same boundary and intersection with $A \cup C \cup B$ as $D$ but has at least one less component of intersection with $K_{1}$ which fails to link $A \cup c \cup B$. This process can be repeated and after doing so a finite number of times a new disk $D$ is found such that each $s_{k}$ in $D \cap K_{1}$ links $A \cup c \cup B$.

That $D \cap K_{1} \neq \square$ follows from the fact that the new $D$, like the old, contains $U \subset$ Int $K_{1}$ and $\partial D \subset$ Ext $K_{1}$, so there is an index $i$ such that $D_{i} \cap K_{1}$ $=s_{i}$. Let $K_{2}=Y_{i} \cup D_{i}$. Since $s_{i}$ links $A \cup c \cup B, Y_{i} \cap C=U_{1}$ and $D_{i} \cap C=U$, so $K_{2} \cap C=U_{1} \cup U$ and $K_{2}$ is the required set if it can be shown to be in $\mathfrak{P}^{*}(T, \epsilon)$. To show this it must first be shown that $\Omega_{2} \in P(T, \epsilon)$, i.e., that $K_{2}$, $T$, and $\epsilon$ satisfy the five conditions of Definition 2.21 .

1. Since $D_{i} \cap Y_{i} \subset D_{i} \cap K_{1}=s_{i}$ and $\partial D_{i}=\partial Y_{i}=s_{i}, K_{2}$ is a topological 2sphere.

2. In order to prove $T \subset$ Int $K_{2}$ the fact that $C \backslash\left(U_{1} \cup U\right)$ has at most three components is needed. To establish this the following more general statement, which will be useful later, is to be proved.

6.11. If $V_{1}$ and $V_{2}$ are disjoint elements of $\mathfrak{T}$, then $C \backslash\left(V_{1} \cup V_{2}\right)$ has at most three components.

For if $V_{1} \in \mathfrak{T}$, then $V_{1}=g\left(v \times E^{k-1}\right)$ for some $g \in \mathfrak{S}$ and $0 \leqq v \leqq 1$. Letting $I_{0}=\left\{(x, y, z) \in E^{k} \mid 0 \leqq x<v\right\}$ and $I_{1}=\left\{(x, y, z) \in E^{k} \mid v<x \leqq 1\right\}$, it is seen that $C \backslash V_{1}$ is the union of $M_{0}=g\left(I_{0} \times E^{k-1}\right)$ and $M_{1}=g\left(I_{1} \times E^{k-1}\right)$. Further, $M_{0} \cup V_{1}$ and $M_{1} \cup V_{1}$ are both $k$-cells if $v$ is neither 0 nor 1 , and since the adjustment needed in the following arguments when $v=0$ or $v=1$ are easily supplied, the assumption $0<v<1$ is made. Since $M_{0}$ and $M_{1}$ are topologically the same it is also assumed without loss of generality that $V_{2} \subset M_{1}$. Then $C \backslash\left(V_{1} \cup V_{2}\right)$ $=\left(C \backslash V_{1}\right) \backslash V_{2}=M_{0} \cup\left(M_{1} \backslash V_{2}\right)$ and if this set has more than three components $M_{1} \backslash V_{2}$ must have more than two, i.e., $M_{1} \backslash V_{2}=N_{1} \cup N_{2} \cup N_{2}$ where $M_{0}, N_{1}, N_{2}$, 
and $N_{3}$ are pairwise disjoint non-null sets and each $N_{i}$ is a union of components of $M_{1} \backslash V_{2}$. But since $V_{2}$ is in $\mathfrak{T}, C \backslash V_{2}$ has at most two components so at least two of the sets $N_{i}$, say $N_{1}$ and $N_{2}$, have closures that meet $M_{0} \cup V_{1}$. Since $\mathrm{Cl} N_{i} \subset \mathrm{Cl} M_{1}=M_{1} \cup V_{1}$, this requires that neither $V_{1} \cap \mathrm{Cl} N_{1}$ nor $V_{1} \cap \mathrm{Cl} N_{2}$ be null. But $M_{1} \cup V_{1}$ is a $k$-cell and $V_{1} \cap V_{2}=\square$, so there is a set $M_{1}^{\prime}$ which is topologically the product of $E^{k-1}$ and an open interval which contains all points of $M_{1}$ in some neighborhood of $V_{1}$. Thus $N_{1}$ and $N_{2}$ both meet a connected subset of $M_{1} \backslash V_{2}$, contradicting the choice of $N_{1}$ and $N_{2}$. This proves 6.11 .

Applying 6.11 to the present situation yields that $C \backslash\left(U_{1} \cup U\right)$ has components $M \cup N \cup R$ and that the two components of $C \backslash U$ are $\mathcal{A}=M \cup U_{1} \cup N$ and $\mathrm{B}=R$, where, it will be recalled, $A$ is the component of $C \backslash U$ containing $U_{1}$ and $T$ while $U_{2} \subset \mathcal{B}$. Since $K_{1} \cap C=U_{1} \cup U_{2}$ and $K_{1}$ separates $T \subset$ Int $K_{1}$ from $A \cap C=a$ in $R^{3}$, it follows that the assumption that $M \cup A \subset$ Ext $K_{1}$ and $T \subset N \subset$ Int $K_{1}$ is but a notational convenience. Since $N$ is connected and does not meet $K_{2}$, if $T \subset$ Ext $K_{2}$ then $N \subset$ Ext $K_{2}$. Since $M \cup A$ is an unbounded subset of the complement of $K_{2}, M \cup A \subset$ Ext $K_{2}$ also. But Ext $K_{2}$ is locally connected and since $M$ and $N$ have common limit points (in $U_{1}$ ), there are arbitrarily small connected sets in the complement of $K_{2}$ meeting both $M$ and $N$. If these sets are sufficiently small they must lie in a neighborhood of $U_{1}$ containing no point of $D$ and hence do not meet $K_{1} \backslash K_{2} \subset D$. This requires that they not meet $K_{1}$ and affords a contradiction since $M \subset$ Ext $K_{1}$ and $N \subset$ Int $K_{1}$.

3. Since both $K_{1}$ and $D$ are locally polyhedral $\bmod C, K_{2} \subset K_{1} \cup D$ must be also.

4. By construction $K_{2} \cap C=U_{1} \cup U$ and both $U_{1}$ and $U$ are in $\mathfrak{T}$.

5. If $p$ is any point of Ext $K_{1}$, then there is a topological ray $J$ from $p$ in Ext $K_{1}$ and $J \cap X_{i} \subset J \cap K_{1}=\square$. But the disk $D_{i}$ is in $K_{1} \cup$ Int $K_{1}$ so $J \cap D_{i}$ $=\square$ also. Hence $J \cap K_{2}=J \cap\left(X_{i} \cup D_{i}\right)=\square$ so $J$ is an unbounded subset of the complement of $K_{2}$ which requires that $J$, and a fortiori $p$, be in Ext $K_{2}$. This proves Ext $K_{1} \subset$ Ext $K_{2}$ so that by complementation in $R^{3}, K_{2} \cup$ Int $K_{2}$ $\subset K_{1} \cup$ Int $K_{1} \subset S(T, \epsilon)$.

Thus $K_{2}$ is in $\mathfrak{B}(T, \epsilon)$ and by the proof of $5, K_{2}$ satisfies condition 1 of Theorem 4.1 also. That the second condition of that theorem is satisfied follows immediately from the hypothesis $U_{1}<T<U$. Thus $K_{2} \in \mathfrak{P}^{*}(T, \epsilon)$.

This completes the proof of the lemma for the case $U_{1}<T<U<U_{2}$, and the only other possible case, $U_{1}<U<T<U_{2}$, can be made to depend on the first case in the following way. Consider the effect upon the order relation and the hypotheses and conclusion of the lemma if the names $A$ and $B$ are interchanged and $h^{*}=h r$ is used instead of $h$, where $r$ is the homeomorphism of $E^{k}$ onto itself defined by $r\left(x_{1}, x_{2}, \cdots, x_{k}\right)=\left(1-x_{1}, x_{2}, \cdots, x_{k}\right)$. Evidently the new $A$ and $B$ are as required since $T_{0}$ and $T_{1}$ are interchanged, and since the order relation is reversed as well as the sets $U_{1}$ and $U_{2}$, the second case is reduced to the first. 
6.2. Definition. For every $T \in \mathfrak{T}_{h}$ and $\epsilon>0$ let $\mathfrak{Q}(T, \epsilon)$ denote the collection of all $K \subset R^{3}$ such that

1. $K$ is a topological 2-sphere.

2. $T \subset$ Int $K$.

3. $K$ is locally polyhedral modulo $C$.

4. $K \cap[A \cup C \cup B]=L \cup R$ where each of the sets $L$ and $R$ is either an element of $\mathfrak{I}$ or a point of $(A \cup B) \backslash C$.

5. $L<T<R$.

6. $K \subset S(C, \epsilon)$.

It should be noted that $\mathfrak{Q}(T, \epsilon)$ depends on $h, A$, and $B$. This dependence is not indicated in the notation since in the applications $h, A$, and $B$ will be chosen and fixed.

6.3. Lemma. Let $0 \leqq x_{1}<x_{2} \leqq 1, T_{x_{i}}=h\left(x_{i} \times E^{k-1}\right), K_{i} \in \mathfrak{Q}\left(T_{x_{i}}, \epsilon\right)$ and $K_{i} \cap(A \cup C \cup B)=L_{i} \cup R_{i}, i=1,2$, be such that $L_{1}<L_{2}<R_{1}<R_{2}$. Then there is a $K_{3}$ in $\mathfrak{Q}\left(T_{x_{1}}, \epsilon\right)$ with $K_{3} \cap(A \cup C \cup B)=L_{1} \cup R_{2}$ and $(A \cup C \cup B) \cap \operatorname{Int} K_{3}$ $=(A \cup C \cup B) \cap\left[\left(\right.\right.$ Int $\left.K_{1}\right) \cup\left(\right.$ Int $\left.\left.K_{2}\right)\right]$.

Proof. As before the adjustments needed for the cases where some or all of the sets $L_{1}, R_{1}, L_{2}, R_{2}$ are points of $(A \cup B) \backslash C$ are easily made, so only the case where all are elements of $\mathfrak{I}$ will be considered. As a preliminary step the following statement is to be proved.

6.31. If $K \in \mathfrak{Q}(T, \epsilon)$ then $(A \cup C \cup B) \cap \operatorname{Int} K=\mathbb{B}(L) \cup \mathcal{A}(R)$.

Since $L<T<R, L$ separates $C \cap A=a$ and $T$ on $C, R$ separates $T$ and $b=B \cap C$, and each separates $a$ and $b$ on $C$, so the three components of $C \backslash(L \cup R)$ as guaranteed by 6.11 must be $C_{A}, C_{B}$, and $C_{T}$; that is, the one containing $a$, the one containing $b$, and the one containing $T$ respectively. Thus $A \cup C \cup B=A \cup C_{A} \cup L \cup C_{T} \cup R \cup C_{B} \cup B$ and since $A \cup C_{A}$ and $C_{B} \cup B$ are unbounded connected sets in the complement of $K$, they lie in Ext $K$. Hence $(A \cup C \cup B) \cap \operatorname{Int} K \subset C_{T}$ and, since $C_{T}$ is connected, does not meet $K$, and contains $T \subset$ Int $K$, the reverse inclusion also holds so

$$
C_{T}=(A \cup C \cup B) \cap \text { Int } K .
$$

Now the two components $\mathcal{A}(L)$ and $\mathrm{B}(L)$ of $(A \cup C \cup B) \backslash L$ are then $A \cup C_{A}$ and $C_{T} \cup R \cup C_{B} \cup B$, and since $L<T$ implies $T \subset \mathcal{B}(L)$ it follows that $A(L)=A \cup C_{A}$ and $B(L)=C_{T} \cup R \cup C_{B} \cup B$. Similarly from $T<R$ it is seen that $A(R)=A \cup C_{A} \cup L \cup C_{T}$ while $\mathscr{B}(R)=C_{B} \cup B$. Thus $A(R) \cap B(L)$ $=\left(A \cup C_{A} \cup L \cup C_{T}\right) \cap\left(C_{T} \cup R \cup C_{B} \cup B\right)=C_{T}$ which proves 6.31.

To proceed with the proof of Lemma 6.3, it is noted that since $L_{1}<L_{2}<R_{1}$ then $L_{2}$ is in both $\mathcal{B}\left(L_{1}\right)$ and $\mathcal{A}\left(R_{1}\right)$ so, by $6.31, L_{2}$ is in Int $K_{1}$ and $K_{2} \cap$ Int $K_{1}$ $\neq \square$. But $R_{1}<R_{2}$ so $R_{2} \subset \mathcal{B}\left(R_{1}\right)$ and, by $6.31, \mathcal{B}\left(R_{1}\right)$ is in Ext $K_{1}$. Thus $K_{2}$ meets Ext $K_{2}$ as well as Int $K_{1}$ and $K_{2} \cap K_{1} \neq \square$ follows. Since $K_{2} \cap K_{1} \cap C$ $=\left(L_{2} \cup R_{2}\right) \cap\left(L_{1} \cup R_{1}\right)=\square$ there is a neighborhood $V$ of $K_{1} \cap K_{2}$ containing no points of $A \cup C \cup B$ and both $K_{1}$ and $K_{2}$ are locally polyhedral at each point of $V$. By shifting the vertices of $K_{2}$ lying in $V$ a distance so small that 
no point outside $V$ is moved, $K_{1}$ and $K_{2}$ may be brought into relative general position so that $K_{1} \cap K_{2}$ is a finite collection of mutually disjoint simple closed curves $s_{1}, s_{2}, \cdots, s_{n}$. Each $s_{j}$ bounds a pair of sub-disks $X_{i j}$ and $Y_{i j}$ of $K_{i}$ where $X_{i j}$ contains $L_{i}, i=1,2$.

Let an arc $c$ be chosen in $C$ so that it has only its end points $a=A \cap C$ and $b=C \cap B$ in common with $\partial C$ and meets each of the sets $L_{1}, L_{2}, R_{1}$, and $R_{2}$ in a single point. Now suppose $s_{k}$ links $A \cup c \cup B$. Then none of the four disks $X_{1 k}, X_{2 k}, Y_{1 k}, Y_{2 k}$ bounded by $s_{k}$ can lie in the complement of $A \cup c \cup B$ so $X_{i k} \supset L_{i}$ and $Y_{i k} \supset R_{i}, i=1,2$. If on the other hand $s_{k}$ does not link $A \cup c \cup B$ then each of the sets $X_{i k} \cap(A \cup c \cup B)$ and $Y_{i k} \cap(A \cup c \cup B)$ must consist of two points or no points for each choice of $i$, and since $L_{i} \subset X_{i k}$ it follows that $R_{i} \subset X_{i k}$ also and $Y_{i k} \cap(A \cup c \cup B)=\square, i=1,2$. No other possibilities exist, since $s_{k}$ either does or does not link $A \cup c \cup B$, so for any $k$ either $R_{i} \subset Y_{i k}$ for both $i=1$ and $i=2$, or $Y_{1 k}$ and $Y_{2 k}$ are both disjoint from $A \cup C \cup B$.

If there is any index $k$ such that $Y_{1 k}$ is disjoint from $A \cup C \cup B$, then there is evidently one such index $j$ such that $Y_{1 j} \cap K_{2}=s_{j}$. Then $Z=Y_{1 j} \cup Y_{2 j}$ is a topological 2-sphere for $Y_{1 j} \cap Y_{2 j}=Y_{1 j} \cap K_{2}=s_{j}$ and $s_{j}=\partial Y_{1 j}=\partial Y_{2 j}$. Further, $Z$ is polyhedral since both $Y_{1 j}$ and $Y_{2 j}$ are subsets of spheres which are locally polyhedral mod $C$ and neither $Y_{1 j}$ nor $Y_{2 j}$ meets $A \cup C \cup B$. Beispeil III, $\S 3$, [5] can be invoked here to produce a semi-linear map of $R^{3}$ onto itself throwing $Y_{1 j}$ onto $Y_{2 j}$ and leaving $A \cup C \cup B$ fixed, but the question of how the interiors of $K_{1}, K_{2}$, and the interiors of their images under this map are related would then arise. To avoid this difficulty an isotopy on $R^{3}$ achieving the above result is to be constructed.

There is a semi-linear homeomorphism $f$ of $R^{3}$ onto itself throwing $Z$ onto the surface of the unit cube $E$ in $R^{3}$ and evidently it may be supposed that if $E^{+}$and $E^{-}$denote the portions of the surface of $E$ on and above and on and below the $x y$-plane respectively, then $f\left(Y_{1 j}\right)=E^{+}$and $f\left(Y_{2 j}\right)=E^{-}$.

An isotopy $g_{t}(0 \leqq t \leqq 1)$ of $R^{3}$ onto itself which throws $E^{-}$onto $E^{+}$and moves no point outside $S(E, \delta)$ with $\delta$ arbitrarily small can be defined with no difficulty, and there is no loss in taking $g_{1}$ semilinear. Then any point of $f(A \cup C \cup B)$ which is interior (exterior) to $f\left(K_{2}\right)$ at the stage $t=0$ remains interior (exterior) to $g_{t} f\left(K_{2}\right)$ for each $t$, so that $f^{-1} g_{1} f\left(K_{2}\right)$ has all the properties required of the original $K_{2}$. The $\delta$ may be taken so small that no point of $f\left(K_{2}\right)$ lies interior to $S(E, \delta)$ except $f\left(Y_{2 j}\right)$ and an arbitrarily small neighborhood of $f\left(Y_{2 j}\right)$ relative to $f\left(K_{2}\right)$, and the isotopy may be defined so as to move no point of $f\left(K_{2}\right)$ except $f\left(Y_{2 j}\right)$.

Consequently $f^{-1} g_{1} f\left(K_{2}\right)$ is $\left(K_{2} \backslash Y_{2 j}\right) \cup Y_{1 j}$ and this sphere may be deformed semi-linearly away from $K_{1}$ in a neighborhood of $Y_{1 j}$ so small that no point of $A \cup C \cup B$ is moved. The resulting sphere is a new $K_{2}$ which has all the properties required of the old and which has at least one less intersection $s_{k}$ with $K_{1}$ which does not link $A \cup c \cup B$.

Thus after a finite number of repetitions a new $K_{2}$ is obtained such that 
every component of its intersection with $K_{1}$ links $A \cup c \cup B$. Then each remaining $X_{1 k}$ contains $L_{1}$ and not $R_{1}$, and an index $p$ is found such that $X_{1 p} \cap K_{2}=s_{p}$. Let $K_{3}=X_{1 p} \cup Y_{2 p}$, and note that $K_{3} \cap(A \cup C \cup B)=\left[X_{1 p}\right.$ $\cap(A \cup C \cup B)] \cup\left[Y_{2 p} \cap(A \cup C \cup B)\right]=L_{1} \cup R_{2}$. Thus in view of $6.31, K_{3}$ is the desired sphere provided it can be shown to satisfy the six conditions of Definition 6.2 for being an element of $\mathfrak{Q}\left(T_{x_{1}}, \epsilon\right)$.

1. Since $X_{1 p} \cap Y_{2 p}=s_{p}=\partial X_{1 p}=\partial Y_{2 p}, K_{3}$ is a topological 2-sphere.

2. The proof that $T_{x_{1}} \subset$ Int $K_{3}$ is essentially a repetition of the argument given to show $T \subset$ Int $K_{1}$ in the proof of Lemma 6.1 and hence need only be outlined here. From $K_{1} \in \mathfrak{Q}\left(T_{x_{1}}, \epsilon\right)$ and $L_{1}<L_{2}<R_{1}<R_{2}$ it is seen that $\mathcal{A}\left(T_{x_{1}}\right) \backslash L_{1}$ has two components, $\mathcal{A}\left(L_{1}\right)$ in Ext $K_{3}$ and $N$ containing $T_{x_{1}}$. If $T_{x_{1}} \subset$ Ext $K_{3}$, then $N \subset$ Ext $K_{3}$ and connected subsets of Ext $K_{3}$ meeting both $N$ and $A\left(L_{1}\right)$ with arbitrarily small diameters exist, for $(\mathrm{Cl} N) \cap \mathrm{Cl} \mathcal{A}\left(L_{1}\right)$ $=L_{1} \neq \square$. But if the diameters of such sets are sufficiently small they are seen to lie in a neighborhood of $L_{1}$ in which $K_{3}$ and $K_{1}$ are identical, and hence lie in the complement of $K_{1}$. This contradicts $T_{x_{1}} \subset$ Int $K_{1}$, which cannot be false for $K_{1} \in \mathfrak{Q}\left(T_{x_{1}}, \epsilon\right)$.

3. $K_{3} \subset K_{1} \cup K_{2}$ and hence is locally polyhedral modulo $C$ for both $K_{1}$ and $K_{2}$ are.

4. $K_{3} \cap(A \cup C \cup B)=L_{1} \cup R_{2}$, and these are as required since they are components of intersection of $A \cup C \cup B$ and $K_{1}$ and $K_{2}$ respectively.

5. That $L_{1}<T_{x_{1}}<R_{2}$ holds was shown above.

6. $K_{3} \subset K_{1} \cup K_{2} \subset S(C, \epsilon)$ as required.

This completes the proof.

\section{The enclosure property.}

7.1. TheOREM. If there is an $h \in \mathfrak{S}$ such that $C$ has property $P$ relative to $\mathfrak{T}_{h}$ and the uniform disk property relative to $\mathfrak{I}_{h}$, then $C$ has the enclosure property.

Proof. Let $\epsilon>0$ be assigned. Let $A^{\prime}$ and $B^{\prime}$ be a pair of topological rays with initial points $a=A^{\prime} \cap C$ in $T_{0} \backslash \partial T_{0}$ and $b=B^{\prime} \cap C$ in $T_{1} \backslash \partial T_{1}$ respectively. That such a pair of rays which are mutually disjoint and locally polyhedral $\bmod C$ exist was shown in the preceeding section. Let $\omega_{1}$ denote min $[\epsilon$, $\left.d\left(T_{0}, T_{1}\right) / 2, d\left(T_{0}, B^{\prime}\right), d\left(T_{1}, A^{\prime}\right)\right]$ and choose $K_{0}$ in $\mathfrak{B}^{*}\left(T_{0}, \omega_{1}\right)$ and $K_{1}$ in $\mathfrak{B}^{*}\left(T_{1}, \omega_{1}\right)$. Let $A^{\prime \prime}$ be the sub-arc of $A^{\prime}$ which is minimal with respect to containing $K_{0} \cap A^{\prime}$. By the choice of $\omega_{1}$ and the fact that $T_{0} \subset \partial C, K_{0} \cap C$ $=R_{0} \in \mathfrak{T}$ and $K_{0} \backslash C$ is an open disk so there is an arc $A^{\prime \prime \prime}$ in $K_{0} \backslash C$ with the same end points as $A^{\prime \prime}$. The arc $A^{\prime \prime \prime}$ may be taken polyhedral and by further subdividing it and shifting all of its vertices except one end point into Ext $K_{0}$ a sufficiently small distance so as to introduce no new points of intersection with $B \cup C \cup K_{1}$ the ray $\left(A^{\prime} \backslash A^{\prime \prime}\right) \cup A^{\prime \prime \prime}$ is deformed into a ray $A$ such that $A \cap K_{1}=\square, A \cap K_{0}=L_{0}$, a point, and $A \cap C=a$, the initial point of $A$. A ray $B$ is constructed from $B^{\prime}$ in a similar manner so that $B \cap K_{0}=\square, B \cap K_{1}=R_{\text {: }}$; a point, and $B \cap C=b$, the initial point of $B$. 
Now $K_{0}$ and $K_{1}$ are seen to be in $\mathfrak{Q}\left(T_{0}, \omega_{1}\right)$ and $\mathfrak{Q}\left(T_{1}, \omega_{1}\right)$ respectively, for all of the six conditions of 6.2 are satisfied by virtue of the fact that $K_{i}$ is in $\mathfrak{B}^{*}\left(T_{i}, \omega_{1}\right), i=0,1$, except condition $4: K_{i} \cap(A \cup C \cup B)=L_{i} \cup R_{i}$ where each of the sets $L_{i}$ and $R_{i}$ is either an element of $\mathfrak{T}$ or a point of $(A \cup B) \backslash C$. But $K_{0} \cap(A \cup C \cup B)=\left(K_{0} \cap A\right) \cup\left(K_{0} \cap C\right) \cup\left(K_{0} \cap B\right)=L_{0} \cup R_{0} \cup \square$ where $L_{0}$ is a point of $A$ and $R_{0}$ is in $\mathfrak{T}$ by construction. Similarly $K_{1} \cap(A \cup C \cup B)=\square \cup L_{1}$ $\cup R_{1}$ where $L_{1}$ is in $\mathfrak{T}$ and $R_{1}$ is a point of $B$.

Since $T_{0} \subset\left(\right.$ Int $\left.K_{0}\right) \cap\left(\right.$ Ext $\left.K_{1}\right)$ and $T_{1} \subset\left(\right.$ Int $\left.K_{1}\right) \cap\left(\right.$ Ext $\left.K_{0}\right)$ it is seen that two real numbers $p, q$ with $0<p<1 / 2<q<1$ can be chosen so that $T_{x} \subset$ Int $K_{0}$ for $0 \leqq x \leqq p$ and $T_{x} \subset$ Int $K_{1}$ for $q \leqq x \leqq 1$. Let such a $p$ and $q$ be chosen and let $M$ denote $\bigcup_{p \leqq x \leqq q} T_{x}$. The plan of proof will be first to show there is a $K_{p}$ in $\mathfrak{Q}\left(T_{p}, \epsilon\right)$ such that $M \subset$ Int $K_{p}$, and then to apply Lemma 6.3 twice, first to $K_{0}$ and $K_{p}$ and then to the resulting sphere and $K_{1}$, to obtain the desired sphere. In order to construct $K_{p}$ some preliminary steps are necessary.

If $\mathfrak{T}_{m}$ denotes the subset of $\mathfrak{I}_{h}$ consisting of all $T_{x}$ in $\mathfrak{I}_{h}$ with $p \leqq x \leqq q$, $\mathfrak{T}_{m}$ is evidently a sub-arc of $\mathfrak{T}_{h}$ and hence is compact. If we let $\omega_{2}$ denote Min $\left[\omega_{1}, d\left(M, T_{0} \cup T_{1} \cup A \cup B\right)\right]$ and $\omega_{3}>0$ be the number corresponding to $\omega_{2}$ guaranteed by the hypothesis that $C$ has the uniform disk property relative to $\mathfrak{T}_{h}$; that is let $\omega_{3}$ be such that if $T_{z} \in \mathfrak{T}_{h}$ and $\eta>0$ there is a $D$ in $\mathfrak{D}\left(T_{z}, \eta\right)$ with $d(\partial D, C)>\omega_{3}$ and $D \subset S\left(T_{2}, \omega_{2}\right)$. Now, since $\mathfrak{T}_{m}$ is compact and $C$ has property $\mathcal{P}$ relative to $\mathfrak{T}_{h}$ (and hence relative to $\mathfrak{I}_{m}$ ), there is a $\gamma>0$ such that for every $T_{x} \in \mathfrak{I}_{m}$, i.e., every $p \leqq x \leqq q$, $\sup _{K \in \mathfrak{P}^{*}\left(T_{x}, \omega_{8}\right)} d\left(T_{x}, K\right)>2 \gamma$. Evidently then there is a $K_{x}$ in $\mathfrak{B}^{*}\left(T_{x}, \omega_{3}\right)$ such that $T_{y} \subset$ Int $K_{x}$ for all $y$ such that $\rho\left(T_{x}, T_{y}\right)<\gamma$. Since $h$ is a homeomorphism, it is easily seen that there is an $\alpha>0$ corresponding to $\gamma$ such that whenever $|x-y| \leqq \alpha$ then $\rho\left(T_{x}, T_{y}\right)<\gamma$. Without loss of generality it may be supposed that $p-\alpha>0$ and $q+\alpha<1$.

Let $\quad x_{1}=p-\alpha, \quad x_{2}=p, \quad x_{3}=p+\alpha, \cdots, \quad x_{i}=p+(i-2) \alpha, \cdots, \quad x_{j+1}$ $=p+(j-1) \alpha$, where $j$ is chosen so that $x_{j}<q \leqq x_{j+1}$. For $i=2,3, \cdots, j$, choose $\Omega_{i}^{\prime}$ in $P^{*}\left(T_{x_{i}}, \omega_{3}\right)$ such that $T_{y} \subset$ Int $K_{i}$ for all $y$ with $x_{i-1} \leqq y \leqq x_{i+1}$. This is possible for if $x_{i-1} \leqq y \leqq x_{i+1}$ then $\left|x_{i}-y\right| \leqq \alpha$ and $\rho\left(T_{x_{i}}, T_{y}\right)<\gamma$.

It will now be shown that from $K_{i}^{\prime}$ a sphere $K_{i}$ in $\mathfrak{B}^{*}\left(T_{x_{i}}, \omega_{3}\right)$ can be formed with the property that $K_{i} \cap C=L_{i} \cup R_{i}$ with $\rho\left(L_{i}, T_{x_{i-1}}\right)$ and $\rho\left(R_{i}\right.$, $\left.T_{x_{i+1}}\right)$ both arbitrarily small, $i=2,3, \cdots, j$. To see this let $\beta>0$ be assigned. For each $i=1, \cdots, j+1$ choose $D_{i} \in D\left(T_{x_{i}}, \beta\right)$ such that $d\left(\partial D_{i}, C\right)>\omega_{3}$ and $D_{i} \subset S\left(T_{x_{i}}, \omega_{2}\right)$. Then $D_{i+1}, K_{i}^{\prime}, T_{x_{i}}$, and $\omega_{3}$ satisfy the seven hypotheses of Lemma 6.1 , as follows.

1. $T_{x_{i}}$ is in $\mathfrak{T}$ and separates $A \cap C$ and $B \cap C$ on $A \cup C \cup B$ since each element of $\mathfrak{T}_{h}$ has these properties.

2. $K_{i}^{\prime} \in \mathfrak{P}^{*}\left(T_{x_{i}}, \omega_{3}\right)$ by choice.

3. Since $\left(K_{i}^{\prime} \cup \operatorname{Int} K_{i}^{\prime}\right) \subset S\left(T_{x_{i}}, \omega_{3}\right)$ and $\omega_{3}<d\left(T_{x_{i}}, A \cup B\right)$, both $A$ and $B$ are separated from $T_{x_{i}}$ by $K_{i}^{\prime} \cap C$ on $A \cup C \cup B$. Hence $C \cap K_{i}^{\prime}$ has two components and consists of a pair of elements $L_{i}^{\prime}$ and $R_{i}^{\prime}$ of $\mathfrak{T}$.

4. From the above, if the notation is properly chosen, $L_{i}^{\prime}$ separates $A$ 
and $T_{x_{i}}$ while $R_{i}^{\prime}$ separates $T_{x_{i}}$ and $B$ on $A \cup C \cup B$. By 2 of Theorem 4.1 and Definition 4.2, $T_{x_{i}}$ separates $L_{i}^{\prime}$ and $R_{i}^{\prime}$ on $A \cup C \cup B$, so $L_{i}^{\prime}<T_{x_{i}}<R_{i}^{\prime}$. Since $T_{y} \subset$ Int $K_{i}^{\prime}$ for all $y$ with $\left|x_{i}-y\right| \leqq \alpha$ and since $x_{i-1}+\alpha=x_{i}=x_{i+1}-\alpha$, $L_{i}^{\prime}<T_{x_{i-1}}<T_{x_{i}}<T_{x_{i+1}}<R_{i}^{\prime}$. Letting $U_{i}$ denote $D_{i} \cap C, i=0,1, \cdots, j+1$, and noting that $\rho\left(U_{i}, T_{x_{i}}\right)<\beta$, it is seen from Lemma 3.1 that if a sufficiently small number is used in place of $\beta$ then $L_{i}^{\prime}<U_{i-1}<T_{x_{i}}<U_{i+1}<R_{i}^{\prime}$.

5. Since $U_{i+1}=D_{i+1} \cap C$, then $\rho\left(U_{i+1}, C \cap D_{i+1}\right)<\omega$ for every $\omega>0$ so $D_{i+1}$ satisfies condition 5 of Definition 2.31 for being in $\mathfrak{D}\left(U_{i+1}, \omega\right)$ for every $\omega>0$. That $D_{i+1}$ also satisfies all the other conditions of 2.31 follows from the fact that $D_{i+1}$ was chosen in $\mathfrak{D}\left(T_{x_{i+1}}, \beta\right)$.

6. Since $D_{i+1} \subset S\left(T_{x_{i+1}}, \omega_{2}\right)$ and $K_{i} \in \mathfrak{P}^{*}\left(T_{x_{i}}, \omega_{3}\right)$, then $D_{i+1} \cup K_{i} \subset S\left(M, \omega_{2}\right)$ for $D_{i+1} \cap C, T_{x_{i+1}}$, and $T_{x_{i}}$ are all in $M$ and $\omega_{2}>\omega_{3}$. But $\omega_{2}<d(M, A \cup B)$ so $\left(D_{i+1} \cup K_{i}\right) \cap(A \cup B)=\square$.

7. By choice of $D_{i+1}, d\left(\partial D_{i+1}, C\right)>\omega_{3}$.

Thus Lemma 6.1 applies so there is a $K_{i}^{\prime \prime}$ in $\mathfrak{B}^{*}\left(T_{x_{i}}, \omega_{3}\right)$ with $K_{i}^{\prime \prime} \cap C$ $=L_{i}^{\prime} \cup U_{i+1}$. In the same way it is verified that $D_{i-1}, K_{i}^{\prime \prime}, T_{x_{i}}$, and $\omega_{3}$ also satisfy the hypothesis of Lemma 6.1 with $L_{i}^{\prime}<U_{i-1}<T_{x_{i}}<U_{i+1}$, so there is a $K_{i}$ in $\mathfrak{P}^{*}\left(T_{x_{i}}, \omega_{3}\right)$ with $K_{i} \cap C=U_{i-1} \cup U_{i+1}, i=2,3, \cdots, j$.

Next it must be shown that $K_{i}$ is in $\mathfrak{Q}\left(T_{x_{i}}, \omega_{3}\right), i=2, \cdots, j$. The first three conditions of Definition 6.2 are satisfied by $K_{i}, T_{x_{i}}$, and $\omega_{3}$ by virtue of $K_{i} \in \mathfrak{B}^{*}\left(T_{x_{i}}, \omega_{3}\right)$, while conditions 4 and 5 follow from the construction. Condition 6 also holds, since $S\left(C, \omega_{3}\right) \supset S\left(T_{x_{i}}, \omega_{3}\right) \supset K_{i}$.

Now Lemma 6.3 is to be applied to $K_{2}$ and $K_{3}$. Since $0<x_{2}<x_{3}=x_{2}+\alpha<1$, and since each $K_{i}$ has been shown to be in $\mathfrak{Q}\left(T_{x_{i}}, \omega_{3}\right)$, Lemma 6.3 applies provided $U_{1}<U_{2}<U_{3}<U_{4}$. But $U_{i}=D_{i} \cap C$ and $\rho\left(T_{x_{i}}, U_{i}\right)<\beta$, so if $\beta$ was taken sufficiently small then $U_{1}<U_{2}<\cdots<U_{j+1}$ follows from $T_{x_{1}}<T_{x_{2}}$ $<\cdots<T_{x_{j+1}}$ and Lemma 3.1. Thus there is a $K_{23}$ in $\mathfrak{Q}\left(T_{x_{1}}, \omega_{3}\right)$ with $K_{23} \cap C=U_{1} \cup U_{4}$ and $(A \cup C \cup B) \cap$ Int $K_{23}=[A \cup C \cup B] \cap\left[\left(\right.\right.$ Int $\left.K_{2}\right) \cup($ Int $\left.K_{3}\right)$ ]. This process is repeated with $K_{23}$ and $K_{4}$ to obtain $K_{24}$, with $K_{24}$ and $K_{5}$ to obtain $K_{25}$, and so on until a sphere $K_{2 j}$ in $\mathfrak{Q}\left(T_{x_{1}}, \omega_{3}\right)$ is obtained with $K_{2 j} \cap C=U_{1} \cup U_{j+1}$ and $(A \cup C \cup B) \cap$ Int $K_{2 j}=(A \cup C \cup B) \cap\left[\left(\right.\right.$ Int $\left.K_{1}\right) \cup \ldots$ $\cup\left(\right.$ Int $\left.\left.K_{j}\right)\right]$. Every point of $M$ lies in a $T_{x}$ for some $x$ with $p \leqq x \leqq q$ and hence with $x_{1} \leqq x \leqq x_{j}$ so $M \subset$ Int $K_{2 j}$.

Thus three spheres have been obtained, first $K_{0}$ in $\mathfrak{Q}\left(T_{0}, \omega_{1}\right)$ with $K_{0} \cap(A \cup C \cup B)=L_{0} \cup R_{0}$ where $L_{0}$ is a point of $A$ and $R_{0}$ separates $T_{p}$ and $T_{q}$ on $A \cup C \cup B$, second $K_{2 j}$ in $\mathfrak{Q}\left(T_{x_{1}}, \omega_{3}\right)$ with $K_{2 j} \cap(A \cup C \cup B)=U_{1} \cup U_{j+1}$ where $U_{1}$ separates $T_{0}$ and $T_{p}\left(=T_{x_{2}}\right)$ on $(A \cup C \cup B)$ and $U_{j+1}$ separates $T_{q}$ (for $\left.q \leqq x_{j+1}\right)$ and $T_{1}$ on $(A \cup C \cup B)$, and third $K_{1}$ in $\mathfrak{Q}\left(T_{1}, \omega_{1}\right)$ with $K_{1}$ $\bigcap(A \cup C \cup B)=L_{1} \cup R_{1}$ where $L_{1}$ separates $T_{p}$ and $T_{q}$ on $(A \cup C \cup B)$ and $R_{1}$ is a point of $B$. Hence $L_{0}<U_{1}<R_{0}<U_{j+1}$ and $L_{0}<L_{1}<U_{j+1}<R_{1}$ so, since $0<p<1$, Lemma 6.3 can be applied, first to $K_{0}$ and $K_{2 j}$ to obtain a $K^{\prime}$ in $\mathfrak{Q}\left(T_{0}, \omega_{1}\right)$ with $K^{\prime} \cap(A \cup C \cup B)=L_{0} \cup U_{j+1}$, and then to $K^{\prime}$ and $K_{1}$ to obtain a $K$ in $\mathfrak{Q}\left(T_{0}, \omega_{1}\right)$ with $K \cap(A \cup C \cup B)=L_{0} \cup R_{1}$. 
Then $(A \cup C \cup B) \cap I n t K$ is the union of those portions of $(A \cup C \cup B)$ which were in the interior of any one of the three spheres $K_{0}, K_{2 j}$, and $K_{1}$. But any point of $C$ lies in $T_{x}$ for some $x$, and hence is interior to $K_{0}, K_{2 j}$, or $K_{1}$ according as $0 \leqq x \leqq p, p \leqq x \leqq q$, or $q \leqq x \leqq 1$. Thus $C \subset$ Int $K$. Also, since $K \in \mathfrak{Q}\left(T_{0}, \omega_{1}\right), K \subset S\left(C, \omega_{1}\right) \subset S(C, \epsilon)$. That $K$ is polyhedral follows from the fact that it is locally polyhedral modulo $C$ and does not meet $C$. This completes the proof, but a fact that will prove useful later should be noted here; i.e., the sphere $K$ meets each of the rays $A, B$ in a single point.

8. The strong enclosure property. For the standard cell $E^{k}$ and a given $\epsilon>0$ it is evident that not only is there a polyhedral 2-sphere $M$ in $S\left(E^{k}, \epsilon\right)$ with $E^{k} C$ Int $M$, but the sphere $M$ may be taken to be the boundary of a convex 3 -cell so that a straight ray meeting $E^{k}$ only at its initial point meets $M$ in a single point. Consequently any tame cell will have the following property.

8.1. Definition. $A$ cell $C$ is said to have the strong enclosure property provided to each $h \in \mathfrak{S}$ there corresponds a pair of disjoint topological rays $A, B$ and a sequence of polyhedral 2-spheres $\left\{M_{i}\right\}$ which meet the following conditions:

1. $A(B)$ meets $C$ only at its initial point $a \in T_{0} \backslash \partial T_{0}\left(b \in T_{1} \backslash \partial T_{1}\right)$.

2. $A \cup B$ is locally polyhedral modulo $C$.

3. $M_{i} \subset S(C, 1 / i)$ and $C \subset$ Int $M_{i}, i=1,2, \cdots$.

4. $M_{i}$ meets each of the rays $A, B$ in a single point, $i=1,2, \cdots$.

Whether or not a cell with the enclosure property can fail to have the strong enclosure property is an unanswered question, although it has already been shown that the sets $A, B$, and $\left\{M_{i}\right\}$ can be chosen satisfying all conditions except possibly condition 4 whenever $C$ has the enclosure property.

8.2. THEOREM. If $C$ is a cell with property $P$, then a necessary and sufficient condition that $C$ have the strong enclosure property is that $C$ have the uniform disk property.

Proof of sufficiency. Let $h \in \mathfrak{W}$ be assigned and choose rays $A_{0}, B_{0}$ satisfying all the conditions of Definition 8.1 except possibly condition 4 . Let $\epsilon_{i}$ be a null sequence of positive numbers, and for each integer $i$ let $T_{0 i}$ be a $(k-1)$ cell such that (1) $T_{0 i} \backslash \partial T_{0 i}$ contains $a=A_{0} \cap C$, (2) $T_{0 i} \subset T_{0}$, and (3) $T_{0 i} \subset S(a$, $\left.\epsilon_{i}\right)$. Also for each $i$ let $T_{1 i}$ be a $(k-1)$-cell with similar relationships with $b=B_{0} \cap C$ and $T_{1}$. Then for each $i$ a homeomorphism $h_{i} \in \mathfrak{S}$ can be chosen so that $h_{i}\left(0 \times E^{k-1}\right)=T_{0 i}$ and $h_{i}\left(1 \times E^{k-i}\right)=T_{1 i}$. (In case $C$ is a 1-cell each $h_{i}$ may be taken to be $h$ itself, for then $T_{0 i}=T_{0}$ and $T_{1 i}=T_{1}$.)

Now for each successive $i$ the cell $C$ has property $P$ relative to $\mathfrak{I}_{h_{i}}$ and the uniform disk property relative to $\mathfrak{T}_{h_{i}}$, so the construction of Theorem 7.1 with $\epsilon_{i}$ used as the $\epsilon$ can be carried out. It is recalled that in this construction the original rays (call them $A_{i-1}$ and $B_{i-1}$ ) were replaced by a new pair (call them $A_{i}$ and $\left.B_{i}\right)$ which had the property that $A_{i} \backslash A_{i-1}\left(B_{i} \backslash B_{i-1}\right)$ was an arc obtained by deforming a sub-arc of a sphere $K_{0}$ in $S\left(T_{0 i}, \epsilon_{i}\right)\left[K_{1}\right.$ in $\left.S\left(T_{1 i}, \epsilon_{i}\right)\right]$ 
an arbitrarily small distance, so that $A_{i} \backslash A_{i-1} \subset S\left(T_{0 i}, \epsilon_{i}\right)$ and $B_{i} \backslash B_{i-1}$ $C S\left(T_{1 i}, \epsilon_{i}\right)$ may be assumed. The result of this construction was a polyhedral sphere (say $\left.M_{i}\right)$ in $S\left(C, \epsilon_{i}\right)$ with $C \subset$ Int $M_{i}$ which met each of the rays $A_{i}, B_{i}$ in a single point. Evidently the sequence $\epsilon_{i}$ can be chosen so that $M_{i} \subset S(C$, $1 / i)$ and also sufficiently small that any sequence $\left\{a_{i}\right\}$ with $a_{i} \in A_{i} \backslash A_{i-1}$ must have limit $a$. To see the latter it is only necessary to note that if $a_{i} \in A_{i} \backslash A_{i-1}$ then $a_{i} \in S\left(T_{0 i}, \epsilon_{i}\right)$ so there is an $a_{i}^{*}$ in $T_{0 i}$ such that $d\left(a_{i}, a\right) \leqq d\left(a_{i}, a_{i}^{*}\right)$ $+d\left(a_{i}^{*}, a\right) \leqq \epsilon_{i}+d\left(a_{i}^{*}, a\right)$. But $d\left(a_{i}^{*}, a\right)<\epsilon_{i}$, for $T_{0 i} \subset S\left(a, \epsilon_{i}\right)$ by choice, so $d\left(a_{i}, a\right) \leqq 2 \epsilon_{i}$. Similarly any sequence $\left\{b_{i}\right\}$ with $b_{i} \in B_{i} \backslash B_{i-1}$ may be supposed to have limit $b$.

At the $i$ th stage of this iterative construction $A_{i}\left(B_{i}\right)$ is formed by replacing a sub-arc $A_{i}^{*}\left(B_{i}^{*}\right)$ of $A_{i-1}\left(B_{i-1}\right)$ by an $\operatorname{arc} A_{i}^{* *}=A_{i} \backslash A_{i-1}\left(B_{i}^{* *}=B_{i} \backslash B_{i-1}\right)$. Let $A=\left[A_{0} \backslash \cup A_{i}^{*}\right] \cup \cup A_{i}^{* *}$ and $B=\left[B_{0} \backslash \cup B_{i}^{*}\right] \cup \cup B_{i}^{* *}$. If the sequence $\left\{\epsilon_{i}\right\}$ is properly chosen, then $A$ and $A_{i}$ are identical on a sub-ray of $A$ which includes all points of $A$ that are separated from $a$ by $A_{i}^{* *}$, so that $A \backslash a$ is topologically a ray with the initial point deleted. This implies that $A$ is a ray with initial point $a$ provided $A=\mathrm{Cl}(A \backslash a)$, and this equality follows from the fact that $\lim a_{i}=a$ for every sequence $\left\{a_{i}\right\}$ with $a_{i} \in A_{i}^{* *}$. Similarly $B$ is a ray.

Thus the sufficiency is established provided $A, B$, and the chosen sequence $\left\{M_{i}\right\}$ satisfy condition 4 of Definition 8.1. But $M_{i} \cap A$ is the same as $M_{i} \cap A_{i}$ provided $A$ and $A_{i}$ are the same exterior to a neighborhood of $a$ which lies in Int $M_{i}$, and this can be guaranteed by choosing $\epsilon_{i+1}$ sufficiently small.

Proof of necessity. Let $h \in \mathfrak{S}$ and $\omega>0$ be assigned. In order to show that $C$ has the uniform disk property it is necessary to exhibit a $\delta>0$ such that for every $T \in \mathfrak{T}_{h}$ and $\epsilon>0$ there is a $D$ in $\mathfrak{D}(T, \epsilon)$ with $d(\partial D, C)>\delta$ and $D \subset S(T$, $\omega)$. First the following statement must be established.

8.21. There is a $\beta>0$ such that if $U$ and $T$ are any pair of elements of $\mathfrak{T}_{h}$ with $U \subset S(T, \beta)$, then $\rho(T, U)<\omega / 2$.

If 8.21 is denied, then for each integer $i$ there is a pair $U_{i}, T_{i}$ of elements of $\mathfrak{T}_{h}$ with $U_{i} \subset S(T, 1 / i)$ and $\rho\left(T_{i}, U_{i}\right) \geqq \omega / 2$. Since $\mathfrak{T}_{h}$ is an arc under the metric $\rho$, it may be assumed that $\left\{T_{i}\right\}$ and $\left\{U_{i}\right\}$, considered as elements of $\mathfrak{T}_{h}$, converge to $T \in \mathfrak{T}_{h}$ and $U \in \mathfrak{T}_{h}$ respectively. Since $\rho$ is a metric, $\rho(T, U)$ $\geqq \omega / 2$, and since $\mathfrak{T}_{h}$ is an arc, $T$ and $U$ must be distinct elements of $\mathfrak{T}_{h}$ and hence disjoint sets in $R^{3}$. Then $\alpha=d(T, U)$ is positive, and by definition of $\rho$, the inclusions $T_{i} \subset S(T, \alpha / 4)$ and $U_{i} \subset S(U, \alpha / 4)$ must hold for all $i$ greater than some fixed $N$. But if $1 / i<\alpha / 4$ and $i>N$, then $U_{i} \subset S\left(T_{i}, \alpha / 4\right)$ and hence $U_{i} \subset S(T, \alpha / 2)$, contradicting $U_{i} \subset S(U, \alpha / 4)$ since these two sets are disjoint. From 8.21 the following will be derived.

8.22. There is an $\eta>0$ such that if $U$ and $T$ are any pair of elements of $\mathfrak{T}_{h}$ and $K \in \mathfrak{P}^{*}(T, \eta)$, then $U \subset$ Int $K$ implies $K \in \mathfrak{B}^{*}(U, \omega)$.

It is evident that no matter what $\eta>0$ is chosen $K$ must satisfy all of the requirements of Definition 2.21 for being in $\mathfrak{P}(U, \omega)$ except possibly $K \subset S$ $(U, \omega)$, and that $U$ must separate the components of $K \cap C$ whenever there 
are two, since $T$ does so and both $U$ and $T$ are in $\mathfrak{T}_{h}$. Hence 8.22 follows if $\eta$ can be chosen so that $(K \cup$ Int $K) \subset S(U, \omega)$ whenever $(K \cup$ Int $K) \subset S(T, \eta)$. Let $\eta=\min (\omega / 2, \beta)$, where $\beta$ is the number required by 8.21 , and suppose $p \in(K \cup$ Int $K) \subset S(T, \eta)$. Then $d(p, U) \leqq d(p, q)+d(q, U)$ for any point $q$ of $T$, so $d(p, U) \leqq \eta+\sup _{q \in T} d(q, U) \leqq \omega / 2+\rho(T, U) \leqq \omega / 2+\omega / 2=\omega$, and 8.22 follows.

Now since $C$ has the strong enclosure property, two rays $A^{\prime}$ and $B^{\prime}$ and a sequence $\left\{M_{i}\right\}$ of polyhedral spheres satisfying. Definition 8.1 can be chosen. Let $\alpha=\min \left[\eta, d\left(T_{0}, T_{1}\right) / 2, d\left(T_{0}, B^{\prime}\right), d\left(A^{\prime}, T_{1}\right)\right]$ and choose $K_{0}$ in $\mathfrak{P}^{*}\left(T_{0}, \alpha\right)$ and $K_{p}$ in $\mathfrak{B}^{*}\left(T_{1}, \alpha\right)$, where the index $p$ will be explained below. The rays $A^{\prime}$ and $B^{\prime}$ are used in the now familiar way to form rays $A$ and $B$ such that $A \cap K_{0}$ is a point, $A \cap K_{p}=\square, B \cap K_{0}=\square$, and $B \cap K_{p}$ is a point. Since $A$ and $B$ are identical with $A^{\prime}$ and $B^{\prime}$ in some neighborhood of $C$, it may be supposed that $A, B$, and $\left\{M_{i}\right\}$ satisfy Definition 8.1 , for deleting a finite number of the $M_{i}$ would make this true.

For each $U \in \mathfrak{T}_{h}$ except $T_{0}$ and $T_{1}, \alpha(U)=\min [\eta, d(U, A \cup B)]$ is positive, and $K(U) \in \mathfrak{B}^{*}(U, \alpha[U])$ can be chosen. Let $M(U)$ denote the set of all $T \in \mathfrak{T}_{h}$ such that $T \subset$ Int $K(U)$, and take $K_{0}=K\left(T_{0}\right)$ and $K_{p}=K\left(T_{1}\right)$. Then the collection of all $M(U)$ is an open covering of the arc $\mathfrak{I}_{h}$ and corresponding to the finite sub-covering which must exist is the collection $K_{0}, K_{1}, \cdots, K_{p}$. [ $K_{0}$ and $K_{p}$ must be present, since $T_{0}$ and $T_{1}$ lie in no $M(U)$ except $M\left(T_{0}\right)$ and $M\left(T_{1}\right)$ respectively, by the choice of $\alpha(U)$.] This collection and the arcs $A$ and $B$ are seen to have the following properties.

1. $A, B$, and $\left\{M_{i}\right\}$ satisfy Definition 8.1 .

2. $A \cap K_{i}=\square$ or $L_{0}$, a point, according as $i>0$ or $i=0$, and $B \cap K_{i}=\square$ or $R_{p}$, a point, according as $i<p$ or $i=p$.

3. $K_{0} \cap K_{p}=\square$ and $K_{i} \cap\left(T_{0} \cup T_{1}\right)=\square$ for all $i$.

4. For each $T$ in $\mathfrak{T}_{h}$ there is an index $i$ such that $T \subset$ Int $K_{i}$, and hence $K_{i} \cup$ Int $K_{i} \subset S(T, \omega)$ by the choice of $\eta$.

Now for each $i=0,1, \cdots, p$ the set $K_{i} \cap(A \cup C \cup B)$ is the union of two components $L_{i}$ and $R_{i}$, and each $L_{i}, R_{i}$ is in $\mathfrak{T}$ except that $L_{0}$ is a point of $A$ and $R_{p}$ is a point of $B$. A collection $\sigma_{0}, \sigma_{1}, \cdots, \sigma_{p}$ of polyhedral simple closed curves can therefore be chosen so that $\sigma_{i}$ separates $L_{i}$ and $R_{i}$ on $K_{i}, i=0,1$, $\cdots, p$. It will be shown that $\delta=d\left(C, \sigma_{0} \cup \ldots \cup \sigma_{p}\right)$ is the number corresponding to the assigned $\omega$ required for the uniform disk property relative to $\mathfrak{T}_{h}$.

For let $T \in \mathfrak{T}_{h}$ and $\epsilon>0$ be assigned. Then an index $j$ can be chosen so that $T \subset$ Int $K_{j}$. Corresponding to $\epsilon$ there is an $\epsilon_{1}>0$ such that if $D$ is in $\mathfrak{D}\left(T, \epsilon_{1}\right)$ then $D$ is also in $\mathfrak{D}(T, \epsilon)$ and $D \cap C \subset$ Int $K_{j}$. It may be assumed that $D \subset$ Int $K_{j}$, for some sub-disk of the original $D$ is in $\mathscr{D}(T, \epsilon)$ and has this property. From the sequence $\left\{M_{i}\right\}$ choose a sphere $M$ which separates $C$ from $\partial D \cup \sigma_{1} \cup \ldots \cup \sigma_{p}$ and meets $A$ and $B$ in the points $a$ and $b$ respectively. Then 
$M$ and $D$ may be taken in relative general position so that $M \cap D$ (which is non-null) is the union of a finite collection of mutually disjoint simple closed curves $s_{1}, \cdots, s_{n}$. Each $s_{i}$ bounds a disk $D_{i}$ on $D$ and a pair of disks $X_{i}$ and $Y_{i}$ on $M$, where $X_{i}$ contains the point $a=M \cap A$. If there is an $i$ for which $D_{i} \cap C=\square$, then the theory of linkages may be used as before to show that $Y_{\boldsymbol{i}} \cap(A \cup C \cup B)=\square$, and $M$ may be replaced by the result of deforming $\left(M \backslash Y_{i}\right) \cup D_{i}$ semi-linearly away from $D$ so as to reduce the number of indices $i$ for which $D_{i} \cap C=\square$. The fact that $\left(D_{i} \cup Y_{i}\right) \cap(A \cup C \cup B)=\square$ is used to guarantee that the new $M$ retains the property $C \subset$ Int $M$. After a finite number of repetitions, each remaining $D_{i}$ contains $D \cap C$, so that they are simply ordered by set inclusion and $D$ can be replaced by $D_{1}$, the minimal remaining $D_{i}$. Then $D_{1} \in \mathfrak{D}(T, \epsilon), D_{1} \subset$ Int $K_{j}$, and $D_{1} \cap M=s_{1}=\partial D_{1}$.

Now $M$ and $K_{j}$ are taken in relative general position and application of the theory of linkages yields the result that any simple closed curves on $K_{j} \cap M$ which bound a sub-disk of $K_{j} \backslash(A \cup C \cup B)$ also bound a sub-disk of $M \backslash(A \cup C \cup B)$. A new $M$ is formed for which the number of such curves of intersection with $K_{j}$ is less than before. It must be noted that $\partial D_{1}$ is a subset of the new $M$ and that $\sigma_{j}$ does not meet it. Repetition removes all such components of $M \cap K_{j}$ so that $M \cap K_{j}$ becomes the union of a finite collection of mutually disjoint simple closed curves $s_{1}^{\prime}, s_{2}^{\prime}, \cdots, s_{m}^{\prime}$, each of which separates $a$ and $b$ on $M$ as well as $L_{j}$ and $R_{j}$ on $K_{j}$. Then $\partial D_{1}=s_{1}$ is interior to $K_{j}$ and lies on $M$, so that $s_{1} \cap s_{i}^{\prime}=\square$ for every choice of $i$ and the curves have a natural order $s_{1}^{\prime}, s_{2}^{\prime}, \cdots, s_{q}^{\prime}, s_{1}, s_{q+1}^{\prime}, \cdots, s_{m}^{\prime}$ induced by set inclusion of the sub-disks of $M \backslash b$ which they bound. By the choice of this order $s_{q}^{\prime}$ and $s_{1}$ together separate $M$ into three components, an open disk containing $a$, an open disk containing $b$, and an open annular $\operatorname{ring} R$ such that $R \cap K_{j}=\square$ and $\mathrm{Cl} R=R \cup s_{q}^{\prime} \cup s_{1}$. Since $s_{1} \subset$ Int $K_{j}, \mathrm{Cl} R$ is contained in $K_{j} \cup$ Int $K_{j}$. Similarly $s_{q}^{\prime}$ and $\sigma_{j}$ bound an annular ring $R^{*}$ on $K_{j}$. Evidéntly $D^{*}=D_{1} \cup R$ $\cup R^{*}$ is a disk which is contained in $K_{j} \cup \operatorname{Int} K_{j}$ and hence in $S(T, \omega)$ and $\partial D^{*}=\sigma_{j}$ so that $d\left(C, D^{*}\right)>\delta$. Thus $D^{*}$ is the required disk provided it can be shown to be in $D(T, \epsilon)$.

To show this it must be verified that $D^{*}, T$, and $\epsilon$ satisfy the six conditions of Definition 2.31. Condition 1 is fulfilled by construction and condition 2 follows from the fact that $\partial D^{*}=\sigma_{j}$ was chosen to separate $L_{j}$ and $R_{j}$, the components of $K_{j} \cap C$, on $K_{j}$. Since $D^{*} \cap C=D_{1} \cap C=D \cap C$, condition 3 is fulfilled. Since $D^{*} \subset\left(D \cup M \cup K_{j}\right)$ and all three of these sets are locally polyhedral modulo $C$, so must $D^{*}$ be. This is condition 4 , and condition 5 follows from $D^{*} \cap C=D \cap C$ and $D \in \mathfrak{D}(T, \epsilon)$. Since there is some neighborhood $U$ of $D^{*} \cap C$ such that $U \cap D^{*}=U \cap D$, condition 6 for $D^{*}$ follows also from $D \in \mathfrak{D}(T, \epsilon)$. This completes the proof.

8.3. Theorem. If $C$ has the strong enclosure property and the disk property, then $C$ has property $P$. 
Proof. Let $T \in \mathfrak{T}$ and $\epsilon>0$ be assigned. Only the case $T \cap \partial C=\partial T$ will be considered since the adjustments to the following argument needed when $T \cap \partial C=T$ are easily made. Therefore there is an $h \in \mathfrak{S}$ and a number $p$ with $0<p<1$ such that $T=h\left(p \times E^{k-1}\right)$. For any pair of numbers $m, n$ with $0<m<p<n<1$, a triple of 1 -cells $I_{0}=\left\{x \in E^{1} \mid 0 \leqq x \leqq m\right\}, I_{1}=\left\{x \in E^{1} \mid n\right.$ $\leqq x \leqq 1\}$, and $I_{2}=\left\{x \in E^{1} \mid m \leqq x \leqq n\right\}$ is defined. This in turn determines a triple of sub-cells of $C$ of dimension $k, C_{i}=h\left(I_{i} \times E^{k-1}\right), i=0,1$, and 2. Evidently $T_{0} \subset C_{0}, T_{1} \subset C_{1}$, and $T \subset C_{2}$, and if $p-m$ and $n-p$ are taken sufficiently small, $C_{2} \subset S(T, \epsilon / 2)$. Now choose another pair of numbers $r, s$ so that $m<r<p<s<n$, and note that $T_{r}$ separates $C_{0}$ and $T$ on $C$ while $T_{s}$ separates $T$ and $C_{1}$ on $C$.

Since $C$ has the disk property a pair of disks $D_{r} \in \mathfrak{D}\left(T_{r}, \beta\right)$ and $D_{s} \in \mathfrak{D}\left(T_{s}, \beta\right)$ may be chosen, and by Lemma 3.1 if $\beta$ is taken sufficiently small then $D_{r}\left(D_{s}\right)$ separates $T$ and $T_{m}\left(T_{n}\right)$ on $C$. This requires that $D_{r}\left(D_{s}\right)$ separate $T$ and $C_{0}\left(C_{1}\right)$ on $C$. Also $R=D_{r} \cap C$ and $S=D_{\boldsymbol{s}} \cap C$ are a pair of elements of $\mathfrak{T}$ which lie in $C_{2}$ and hence it may be supposed that $D_{r} \cup D_{s}$ is in $S(T, \epsilon)$, since this can be assured by taking sub-disks of the original ones.

A pair of rays $A, B$, and a sequence of spheres $\left\{M_{i}\right\}$ satisfying Definition 8.1 are now chosen, and again taking sub-disks of $D_{r}$ and $D_{s}$ as new disks $D_{r}$, $D_{s}$ the relation $\left(D_{r} \cup D_{s}\right) \cap(A \cup B)=\square$ can be made to hold. Now let $\delta$ be $\min \left[\epsilon / 2, d\left(C, \partial D_{r} \cup \partial D_{s}\right)\right]$ and choose a polyhedral 2-sphere $M$ from the sequence $\left\{M_{i}\right\}$ so that $M$ is in $S(C, \delta), C \subset$ Int $M$, and $M$ meets $A$ and $B$ in a single point each. Taking $M$ and $D_{r} \cup D_{s}$ in relative general position, using the theory of linkages, and replacing sub-disks of $M \backslash(A \cup B)$ by sub-disks of $\left(D_{r} \cup D_{s}\right) \backslash C$, a new $M$ is formed such that each component of $M \cap\left(D_{r} \cup D_{s}\right)$ is a simple closed curve separating $a=M \cap A$ and $b=M \cap B$ on $M$. Since $R$ separates $a$ and $S$ on $C$, if the $\delta$ above was chosen sufficiently small then $D_{r}$ separates $a$ and $D$, on $S(C, \delta)$ and hence on $M$. But this requires that some component $s_{i}$ of $D_{r} \cap M$ separate $a$ and $D_{s} \cap M$ on $M$ and hence a simple closed curve $s_{j}$ of $D_{r} \cap M$ can be found such that if $X_{j}$ is the sub-disk of $M \backslash D_{\boldsymbol{*}}$ bounded by $s_{j}$ then $X_{j} \cap D_{r}=s_{j}$. Let $M_{0}^{*}$ denote the 2 -sphere which is the union of $X_{j}$ and the sub-disk of $D_{r}$ bounded by $s_{j}$. That $C_{0} \subset$ Int $M_{0}^{*}$ is readily established, for $C_{0} \subset$ Ext $M_{0}$ leads to a contradiction. Similarly a 2 -sphere $M_{1}^{*}$ with $C_{1} \subset$ Int $M_{1}$ is formed from $M$ and $D_{s}$.

Now let $\eta>0$ be chosen less than $\epsilon / 2$ and so that $S\left(C_{0}, \eta\right) \subset$ Int $M_{0}^{*}$, $S\left(C_{1}, \eta\right) \subset$ Int $M_{1}^{*}$, and $S(C, \eta) \cap\left(M_{0}^{*} \cup M_{1}^{*}\right)=S(C, \eta) \cap\left(D_{r} \cup D_{s}\right)$. Choose from the sequence $\left\{M_{i}\right\}$ a 2 -sphere $N$ with $N \subset S(C, \eta)$ such that $C \subset$ Int $N$ and $N$ meets each of the sets $A$ and $B$ in a single point. As before, it may be assumed that each component of $N \cap\left(D_{r} \cup D_{s}\right)=N \cap\left(M_{0}^{*} \cup M_{1}^{*}\right)$ is a simple closed curve separating $N \cap A$ and $N \cap B$ on $N$. These simple closed curves $s_{1}, s_{2}, \cdots, s_{n}$ bound sub-disks $X_{1}, X_{2}, \cdots, X_{n}$ of $N \backslash B$ and since the $s_{i}$ are disjoint and each $X_{i}$ contains $N \cap A$, it may be assumed that $X_{1} \subset X_{2} \subset \cdots$ $\subset X_{n}$. Some of the $s_{i}$ are on $D_{r}$ and some are on $D_{s}$, so an index $j$ can be found 
such that $s_{j}$ lies on one of these two sets (say on $D_{r}$ ) while $s_{j+1}$ lies on the other. The pair $s_{j}, s_{j+1}$ bound an annular ring $R_{j}$ on $N$ and $s_{j}$ bounds a disk $D_{r j}$ on $D_{r}$ while $s_{j+1}$ bounds a disk $D_{s j}$ on $D_{s}$. Let $K=D_{r j} \cup R_{j} \cup D_{s j}$. That $K$ is a topological 2-sphere which is locally polyhedral modulo $C$ follows from the construction, as does the fact that $K \cap C=R \cup S$ where both $R$ and $S$ are in $T$. Thus $\Re$ is in $\mathfrak{B}(T, \epsilon)$ provided $T \subset$ Int $K$ and $K \subset S(T, \epsilon)$.

To see that the latter holds, it is noted first that $D_{r j} \cup D_{s j} \subset D_{r} \cup D_{s} \subset S(T$, $\epsilon / 2)$, so $K \subset S(T, \epsilon)$ if $R_{j} \subset S(T, \epsilon)$. Suppose there is a point $p$ of $R_{j}$ such that $d(p, T) \geqq \epsilon$. Since $R_{j} \subset N \subset S(C, \eta)$ and $\eta<\epsilon / 2$, there is a point $q$ of $C$ such that $d(p, q)<\epsilon / 2$. That $q \in C_{2}$ cannot be, for then $d(p, T) \leqq d(p, q)+d(q, T)<\epsilon$, so $q \in C_{i}$ for $i=0$ or $i=1$. But $S\left(C_{i}, \eta\right) \subset \operatorname{Int} M_{i}^{*}$ so this requires $p \in \operatorname{Int} M_{i}^{*}$ for one choice of $i$, say $i=0$. The choice of $M_{0}^{*}$ and $M_{1}^{*}$ is seen to assure that $M_{0}^{*} \subset$ Ext $M_{1}^{*}$ and $M_{1}^{*} \subset$ Ext $M_{0}^{*}$, and since $R_{j} \cap\left(M_{0}^{*} \cup M_{1}^{*}\right)=s_{j} \cup s_{j+1}=\partial R_{j}$, $R_{j} \backslash\left(s_{j} \cup s_{j+1}\right)$ lies in $\left(\right.$ Ext $\left.M_{0}^{*}\right) \cap\left(\right.$ Ext $\left.M_{1}^{*}\right)$. Hence $R_{j} \cap$ Int $M_{0}^{*}=\square$ and $p \in$ Int $M_{0}^{*}$ is a contradiction, proving that $K \subset S(T, \epsilon)$.

It follows from the arguments above that Int $K$ and Int $M_{i}^{*}$ are disjoint for $i=0$ and $i=1$. Hence the components $C_{0}^{\prime}$ and $C_{1}^{\prime}$ of $C \backslash K$ determined by $C_{0}$ and $C_{1}$ are in Ext $K$. If $T \subset$ Ext $K$ then the third component $C_{T}$ of $C \backslash K$ is also in Ext $K$ and a contradiction is reached just as in the proof of Lemma 6.1 .

Thus since $\epsilon$ and $T$ were arbitrary and $K \in \mathfrak{B}(T, \epsilon)$ has been found, it fóllows that $C$ has property $P$.

9. Conclusion. Theorems 8.2 and 8.3 combine to give the following result.

9.1. ThEOREM. If $C$ is a $k$-cell in $R^{3}$ for $k=1,2$, or 3 , and has any two of the following three properties, then it also has the third.

1. Property $P$.

2. The uniform disk property.

3. The strong enclosure property.

9.2. TheoRem. If $C$ is a 1-cell in $R^{3}$ with property $P$, then $C$ has the strong enclosure property.

If the word strong is deleted here, this becomes a restatement of Theorem 1 of Harrold [6]. The proof given by Harrold, together with the proof of suffciency in Theorem 8.2, establish 9.2 as stated.

9.3. CoRollary. If $C$ is a 1-cell : vith property $P$, then $C$ has the uniform disk property.

Example 1.1 of Fox-Artin [4] is a 1-cell which can be shown to have the uniform disk property but not the enclosure property, and hence of course, not property $\mathcal{P}$. Whether or not a 2 -cell or a 3-cell with property $\mathcal{P}$ can fail to have the enclosure property and/or the uniform disk property is an unanswered question. 


\section{BIBLIOGRAPHY}

1. J. W. Alexander, On the subdivision of space by a polyhedron, Proc. Nat. Acad. Sci. U.S.A. vol. 10 (1924) pp. 6-8.

2. - An example of a simply connected surface bounding a region which is not simply connected, Proc. Nat. Acad. Sci. U.S.A. vol. 10 (1924) pp. 8-10.

3. P. Alexandroff and H. Hopf, Topologie, Berlin, Springer, 1935.

4. R. H. Fox and E. Artin, Some wild cells and spheres in three dimensional space, Ann. of Math. vol. 49 (1948) pp. 979-990.

5. W. Graueb, Die semilinearen Abbildungen, Sitzungsberichte der Heidelberger Akademie der Wissenschaften-Naturwissenschaftliche Klasse, 1950, pp. 205-272.

6. O. G. Harrold, Jr., The enclosing of simple arcs and curves by polyhedra, Duke Math. J. vol. 21 (1954) pp. 615-622.

7. O. G. Harrold, Jr. and E. E. Moise, Almost locally polyhedral spheres, Ann. of Math. vol. 57 (1953) pp. 575-578.

8. F. Hausdorff, Mengenlehre, New York, Dover, 1944.

9. E. E. Moise, Affine structures in 3-manifolds, IV, Ann. of Math. vol. 55 (1952) pp. 215222.

10. A. Schoenflies, Die Entwickelung der Lehre von den Punktmannigfaltigkeiten, Leipzig, Teubner, 1908.

11. R. L. Wilder, Topology of manifolds, Amer. Math. Soc. Colloquium Publications, vol. 32.

UNiversity OF TenNessee,

KNOXVILLE, TENN.

UNIVERSITY OF CONNECTICUT, Storrs, Conn. 